JOURNAL OF THE

AMERICAN MATHEMATICAL SOCIETY

Volume 17, Number 2, Pages 243-265

S 0894-0347(04)00453-9

Article electronically published on February 11, 2004

\title{
ON NEUMANN EIGENFUNCTIONS IN LIP DOMAINS
}

\author{
RAMI ATAR AND KRZYSZTOF BURDZY
}

\section{IntRodUCTION AND MAIN RESUlT}

A planar set $D$ will be called a lip domain if it is Lipschitz, open, bounded, connected, and given by

$$
D=\left\{\left(x_{1}, x_{2}\right): f_{1}\left(x_{1}\right)<x_{2}<f_{2}\left(x_{1}\right)\right\},
$$

where $f_{1}, f_{2}$ are Lipschitz functions with constant 1 . The assumption that $D$ is a Lipschitz domain puts an extra constraint on the functions $f_{k}$; we discuss this issue in greater detail later in this section.

Let $\mu_{2}$ denote the second eigenvalue for the Laplacian in $D$ with Neumann boundary conditions. Here is our main result.

Theorem 1. (i) The second eigenvalue $\mu_{2}$ is simple in all lip domains except squares.

(ii) ("Hot spots conjecture") For every lip domain, every Neumann eigenfunction corresponding to $\mu_{2}$ attains its maximum and minimum at boundary points only.

The "hot spots" conjecture was proposed by J. Rauch at a conference in 1974 and was never published in print so it is somewhat vague; roughly speaking, it says that in Euclidean domains, the second Neumann eigenfunction attains its maximum at the boundary (see [3] for a discussion of different versions of the conjecture). It was shown by Kawohl ([16]) that the conjecture holds in cylinders. Bañuelos and Burdzy ([3]) proved the conjecture for a large class of lip domains and some convex domains. However, their techniques were limited to polygonal domains. In the proof of Theorem 1 (ii), we will show how one can drop this assumption using the results of Burdzy and Chen ([8]). Bañuelos and Burdzy ([3]) were able to prove the "hot spots" conjecture for only one of the eigenfunctions corresponding to $\mu_{2}$. This naturally lead to the problem of characterization of those lip and convex domains where $\mu_{2}$ is simple. They gave a partial answer in [3] -it was shown that the eigenvalue is simple in a convex domain if the ratio of length to width of the domain is larger than 3.07. The critical ratio is only 1.53 if the convex domain is assumed in addition to have a line of symmetry.

Received by the editors December 17, 2001.

2000 Mathematics Subject Classification. Primary 35J05; Secondary 60H30

Key words and phrases. Neumann eigenfunctions, reflected Brownian motion, couplings, hot spots problem.

Research partially supported by the fund for the promotion of research at the Technion.

The second author gratefully acknowledges the hospitality and financial support of Technion (Israel) and Institut Mittag-Leffler (Sweden). This research was partially supported by NSF Grant DMS-0071486 and ISF Grant 12/98. 
The "hot spots" conjecture does not hold in arbitrary planar domains ([10], [5]) but it is still an open problem as to whether it holds in convex domains. See [2], [14] and [20] for some recent results on this problem.

Our main result solves two problems posed by Jerison and Nadirashvili ([14]).

Corollary 1. The following conjectures are true.

(i) (14, Conj. 8.2) If $D$ is a convex polygon with two axes of symmetry and a right angle, then the only case in which the eigenvalue is multiple is when $D$ is a square.

(ii) (14], Conj. 8.5) Let $D$ be a convex, centrally symmetric domain contained in a circular sector of acute angle and vertex $P$. If $P \in \partial D$, then the maximum or the minimum of the eigenfunction corresponding to $\mu_{2}$ is achieved at $P$, and $\mu_{2}$ is simple.

Our proof of Theorem 1 uses a probabilistic technique called "coupling." Two large sections of the article are devoted to the construction (Section 2) and analysis (Section 3) of "mirror couplings" of reflected Brownian motions. Mirror couplings (without reflection) were used by Kendall ([17]) to obtain analytic estimates. The same couplings for reflected diffusions were applied in [22, [3] and 9]. Unfortunately, none of the last three quoted papers paid sufficient attention to the construction of mirror couplings. The paper by Wang [22] does not offer any proof of existence of mirror couplings for reflected diffusions. The papers by Bañuelos and Burdzy [3] and Burdzy and Kendall [9] give an explicit construction for the mirror coupling of Brownian motions reflected on a straight line. This construction breaks down in polygonal domains when two Brownian particles hit two different line segments on the boundary at the same time (proving that this event has a positive probability is nontrivial; we omit the proof as it is not needed here). Although some readers may find our construction of mirror couplings in piecewise $C^{2}$ domains exceedingly detailed, we believe that the history of the problem justifies the extra care we give to it.

The most challenging part of our paper is a probabilistic version of the "parabolic boundary Harnack principle," i.e., Section 3 We will show that if two mirror coupled Brownian motions are conditioned on not meeting by a certain time, then they are likely to be far apart at that time. This intuitively obvious statement seems to have no easy proof. The core of our argument is taken from [4]. The same approach has already been used in [1] and 9 but each application of the same basic idea needs new estimates and some minor changes. The present application of the argument from [4] needs completely new estimates for a few fundamental quantities - this is what makes that part of the paper tedious. An additional complication stems from the fact that our construction of mirror couplings of reflected Brownian motions is limited to piecewise $C^{2}$ domains. We have to consider a sequence of mirror couplings in a sequence of piecewise smooth domains approximating the lip domain. Known results on the existence of strong solutions to the Skorohod equation indicate that the construction of a mirror coupling with the strong Markov property in an arbitrary lip domain may be a very difficult task - we do not try to undertake it in this article.

The proof of the main theorem, using ideas of Atar [2], is given in Section 4 The core argument is the following. Let $\left(e_{1}, e_{2}\right)$ be the usual orthonormal basis for $\mathbb{R}^{2}$. It was observed in [3] (see also Section 4 in this article) that in every lip domain, there exists an "increasing" Neumann eigenfunction corresponding to 
$\mu_{2}$, i.e., an eigenfunction whose directional derivatives in directions $e_{1}+e_{2}$ and $e_{1}-e_{2}$ are nonnegative on $D$. We prove that if $\mu_{2}$ is not simple, then there exists a corresponding eigenfunction which is "increasing" in a large part of the domain but not at all points. The key estimate, Lemma 6 shows that if this condition is satisfied by the gradient in the bulk of the domain, then it has to be satisfied everywhere in the domain. In other words, Lemma 6 provides a link between the local and global estimates for the direction of the gradient of an eigenfunction - this is where the results from Sections 2 and 3 on mirror couplings are used in a crucial way.

We end this introduction with a few remarks on lip domains. This class of domains appeared for the first time in [3] and [9]. The name "lip domains" was coined in 7 .

It follows easily from the definition of a lip domain $D$ that there exist leftmost and rightmost extreme points $x^{*}, y^{*} \in \bar{D}$, i.e., for every $x=\left(x_{1}, x_{2}\right) \in \bar{D} \backslash\left\{x^{*}, y^{*}\right\}$, we have $x_{1}^{*}<x_{1}<y_{1}^{*}$. Recall that the definition of a lip domain $D$ involves a requirement that $D$ be a Lipschitz domain. This means that every $x \in \partial D$ has a neighborhood $U$ such that $\partial D \cap U$ is the graph of a Lipschitz function in some orthonormal coordinate system. Hence, $f_{2}\left(x_{1}\right)-f_{1}\left(x_{1}\right)$ cannot go to 0 very fast as $x_{1} \downarrow x_{1}^{*}$ or $x_{1} \uparrow y_{1}^{*}$. Without this condition, one could construct domains satisfying (11) and containing infinite sequences of "rooms and passages" close to $x^{*}$ and $y^{*}$. The results of [13] indicate that such domains might not have discrete spectrum. The exclusion of these domains from our consideration does not seem to be an essential limitation because lip domains with shapes significantly different from the square are not likely to have multiple second eigenvalues.

We will write $B_{r}(x) \subset \mathbb{R}^{2}$ to denote an open ball of radius $r$ around $x$ and we will use $c$ to denote positive constants whose values are unimportant and may change from line to line.

\section{Construction of Mirror COUplings}

Before we go into technical proofs, we briefly and informally describe the goal of this section. Suppose for a moment that $D \in \mathbb{R}^{d}, d \geq 2$, is a smooth domain. We are looking for a pair of reflected Brownian motions $\left(X_{t}, Y_{t}\right)$ satisfying the system of stochastic differential equations

$$
d X=d W+d L, \quad d Y=d Z+d M, \quad d Z=d W-2 m m \cdot d W, \quad m=\frac{Y-X}{|Y-X|}
$$

for times less than $\zeta=\inf \left\{s: X_{s}=Y_{s}\right\}$. Here $W$ is a $d$-dimensional Brownian motion, and $Z$ is another Brownian motion for which the increments are mirror images of those of $W$, the mirror being the $(d-1)$-dimensional hyperplane with respect to which $X$ and $Y$ are symmetric. The processes $X$ and $Y$ are reflecting Brownian motions with boundary terms $L$ and $M$, respectively. We will prove pathwise uniqueness and strong existence for this system of equations. As a corollary, it will be proved that $\left(X_{t}, Y_{t}\right)$ is strong Markov. The final proposition in this section will show that mirror couplings in lip domains have an "order preserving" property which is crucially used in this paper.

An SDE with reflecting boundary conditions. The starting point for our analysis is some results from [19] regarding unique solvability of an SDE with reflection. The 
results in that paper apply to a class of domains satisfying complicated assumptions - we refer the reader to [19] for details. It will suffice to say that the results of [19] apply to all $D \subset \mathbb{R}^{d}, d \geq 3$, which are $C^{2}$-smooth and all planar piecewise $C^{2}$-smooth domains with a finite number of convex corners (more precisely, domains for which the boundary consists of finitely many smooth parts and for which the uniform exterior sphere condition and the uniform interior cone condition are satisfied; see [19]) . All results in this section hold true for all domains which satisfy the conditions listed in [19, so they are not limited to the cases we have just listed.

Fix a bounded open set $D \in \mathbb{R}^{d}$ for some $d \geq 2$ and assume that its boundary is $C^{2}$-smooth if $d \geq 3$ or piecewise smooth with a finite number of convex corners if $d=2$. For $x \in \partial D$, let $\nu(x)$ be the inward unit normal vector, if it exists. If $x$ is a corner, we let $\nu(x)$ be an arbitrary unit vector pointing inside the domain at $x$ (this is just for the sake of completeness of the definition - the reflected Brownian motion does not visit corners with probability 1 so the definition of $\nu(x)$ for such points is irrelevant).

Let $\left(\Omega, F,\left(F_{t}\right), P\right)$ be a complete filtered probability space, on which an $\left(F_{t}\right)$ Brownian motion $\left(W_{t}\right)$ is given. It was shown in 19 that for any $x \in \bar{D}$ there exists a unique continuous $\left(F_{t}\right)$-semimartingale $\left(X_{t}\right)$ with values in $\bar{D}$ for all $t \geq 0$ a.s., satisfying

$$
X_{t}=x+W_{t}+L_{t}, \quad|L|_{t}=\int_{0}^{t} 1_{X_{s} \in \partial D} d|L|_{s}, \quad L_{t}=\int_{0}^{t} \nu\left(X_{s}\right) d|L|_{s},
$$

where $L_{t}$ is a continuous process with values in $\mathbb{R}^{d}$, with variation $|L|_{t}$ which is bounded on each finite interval. In fact, there is a map $\Gamma: C\left([0, \infty): \mathbb{R}^{d}\right) \rightarrow$ $C\left([0, \infty): \mathbb{R}^{d}\right)$ (the "Skorohod map") such that $X=\Gamma(x+W)$ a.s. Moreover, for every $T>0$, there exists a map $\Gamma_{T}$ from $C\left([0, T]: \mathbb{R}^{d}\right)$ into itself, such that

$$
\left.X\right|_{[0, T]}=\left.\Gamma(x+W)\right|_{[0, T]}=\Gamma_{T}\left(x+\left.W\right|_{[0, T]}\right),
$$

and $\Gamma_{T}$ is Hölder continuous of order $1 / 2$ on compact subsets of $C\left([0, T]: \mathbb{R}^{d}\right)$. This implies that the function $x \mapsto \Gamma_{T}\left(x+\left.W\right|_{[0, T]}\right)$ is continuous. With an abuse of notation, we will refer below to $\Gamma_{T}$ as $\Gamma$. We will call the process $X$ a reflected Brownian motion in $D$ (with normal reflection), driven by $W$, starting from $x$.

Denote

$$
\|Z\|_{t}=\left[E \sup _{0 \leq s \leq t}\left|Z_{s}\right|^{4}\right]^{1 / 4} .
$$

Let $\mathbf{Z}$ be the space of continuous adapted processes $Z$ for which $\|Z\|_{t}<\infty$ for all $t>0$. We shall need the following version of Lemma 3.1 of [19].

Lemma 1. Let $\sigma$ be an $\left(F_{t}\right)$-stopping time. Then for each $T>0$ there exists $C$ such that for all $t \leq T$ and all $Z, Z^{\prime} \in \mathbf{Z}$,

$$
\left\|\Gamma(Z) \cdot \wedge \sigma-\Gamma\left(Z^{\prime}\right) \cdot \wedge \sigma\right\|_{t}^{4} \leq C \int_{0}^{t}\left\|Z \cdot \wedge \sigma-Z_{\cdot \wedge \sigma}^{\prime}\right\|_{s}^{4} d s .
$$

Proof. The only difference between our lemma and Lemma 3.1 of [19] is that the processes are stopped at a stopping time $\sigma$ in our version of the inequality. The original proof given in [19] applies, with minor adjustments needed to take into account the presence of the stopping time $\sigma$. 
Equations for the mirror coupling. We will formulate a system of stochastic differential equations for a pair of "mirror coupled" reflecting Brownian motions, i.e., satisfying the following condition. On any time interval $[s, t]$, on the event $A$ that both processes do not hit the boundary during $[s, t]$, there is a $(d-1)$-dimensional hyperplane (the "mirror"), depending on $\omega \in A$ and $s, t$, with respect to which the processes are symmetric at every time in $[s, t]$.

Let $\left(\Omega, F,\left(F_{t}\right), P, W\right)$ be given, where $W$ is an $\left(F_{t}\right)$-Brownian motion. We are looking for processes $Z, X$ and $Y$ with the following properties. The processes $X$ and $Y$ are reflecting Brownian motions in $D$, starting from $x$ and $y$, and driven by $W$ and $Z$, respectively. Here $Z$ is another Brownian motion on the same probability space. We will work with deterministic initial conditions, but we note that replacing them with $F_{0}$-measurable initial conditions would add no difficulty. We want to have

$$
X=\Gamma(x+W)
$$

as in (2). We also need,

$$
Y=\Gamma(y+Z) .
$$

Let $m_{t}$ be a unit vector perpendicular to the mirror with respect to which $X_{t}$ and $Y_{t}$ are symmetric, namely $m_{t}=\left(Y_{t}-X_{t}\right) /\left|Y_{t}-X_{t}\right|$. Here and later, all vectors will be column vectors. For $m$ in the unit sphere of $\mathbb{R}^{d}$, let $H(m)$ denote the $d \times d$ matrix

$$
H(m)=I-2 m m^{\prime}
$$

where $m^{\prime}$ denotes the transpose of $m$. Then $H(m) v=v-2(m \cdot v) m$ is the mirror image of $v$ about the hyperplane through the origin, perpendicular to $m$. We would like $Z$ to depend on $W$ and on the mirror, in such a way that, before $X$ and $Y$ first meet, $Z$ 's increments are mirror images of $W$ 's:

$$
Z_{t}=\int_{0}^{t} H\left(m_{s}\right) d W_{s}
$$

For $x \in \mathbb{R}^{d}$, let $G(x)=H(x /|x|)$ if $x \neq 0$, and let $G(0)=0$ (the value of $G(0)$ is in fact irrelevant). Equip the space of $d \times d$ matrices with the norm $\|A\|^{2}=\sum_{i, j} A_{i, j}^{2}$. Then it is easy to check that for $|m|=|\widetilde{m}|=1$,

$$
\|H(m)-H(\widetilde{m})\| \leq c|m-\widetilde{m}| .
$$

Consider the equation

(8)

$Z_{t}=\int_{0}^{t \wedge \zeta} G\left(\Gamma(y+Z)_{s}-X_{s}\right) d W_{s}+1_{t \geq \zeta}\left(W_{t}-W_{\zeta}\right), \quad \zeta=\inf \left\{s: \Gamma(y+Z)_{s}=X_{s}\right\}$.

Definition 1. (i) We say that pathwise uniqueness holds for (8) if whenever $\left(\Omega, F,\left(F_{t}\right), P\right)$ is a filtered probability space, $W$ is an $\left(F_{t}\right)$-Brownian motion, $X=$ $\Gamma(x+W)$, processes $Z$ and $Z^{\prime}$ are $\left(F_{t}\right)$-adapted and have continuous sample paths, and both $Z$ and $Z^{\prime}$ satisfy (8) for $t \geq 0$ a.s., then $Z(t)=Z^{\prime}(t)$ for all $t \geq 0$, a.s.

(ii) A strong solution to (8) on a given probability space $(\Omega, F, P)$ relative to the Brownian motion $W$ is a process $Z$ with continuous sample paths, adapted to the (augmented) filtration $\left(F_{t}^{W}\right)$, generated by $W$, and with $X=\Gamma(x+W)$, which satisfies (8) for all $t \geq 0$, a.s. 
Theorem 2. Pathwise uniqueness holds for (8). Let $W$ be a Brownian motion on a complete probability space $(\Omega, F, P)$. Then there exists a strong solution of (8) relative to $W$.

We will refer to the pair $(X, Y)$ as a mirror coupling of reflecting Brownian motions.

Proof of Theorem 2 Pathwise uniqueness. We will first consider the problem on a finite time interval $\left[0, \overline{T]}\right.$. Let a Brownian motion $W$ on $\left(\Omega, F,\left(F_{t}\right), P\right)$ be given, and let $X=\Gamma(x+W)$. Assume $Z$ and $\widetilde{Z}$ are two $\left(F_{t}\right)$-adapted processes with continuous sample paths, satisfying (8) for all $t \in[0, T]$ a.s. Denote $Y=\Gamma(y+Z), \widetilde{Y}=\Gamma(y+\widetilde{Z})$, $V=Y-X, \widetilde{V}=\widetilde{Y}-X$. Let $\tau_{n}=\inf \left\{t:\left|V_{t}\right| \leq 1 / n\right\}, \widetilde{\tau}_{n}=\inf \left\{t:\left|\widetilde{V}_{t}\right| \leq 1 / n\right\}$, $S_{n}=\tau_{n} \wedge \widetilde{\tau}_{n}$. Then $S_{n} \uparrow \zeta \wedge \widetilde{\zeta}$. To prove pathwise uniqueness, it is enough to show that $P\left(Z_{t}=\widetilde{Z}_{t}, 0 \leq t<\zeta \wedge \widetilde{\zeta}\right)=1$. On $\left[0, S_{n}\right]$, one has

$$
\left|\frac{V}{|V|}-\frac{\widetilde{V}}{|\widetilde{V}|}\right| \leq c \frac{|V-\widetilde{V}|}{|V| \wedge|\widetilde{V}|} \leq c n|V-\widetilde{V}|=c n|Y-\widetilde{Y}| .
$$

We will use the following version of the Burkholder-Davis-Gundy inequality ([15. p. 163),

$$
E\left|\int_{0}^{T} A_{t} d W_{t}\right|^{2 m} \leq c_{m} T^{m-1} E \int_{0}^{T}\left|A_{t}\right|^{2 m} d t
$$

for $m \geq 1$ and $A_{t}$ adapted. Since $Z$ is adapted, it follows from (3) that so is the integrand in (8), and therefore $Z$ is a martingale. Doob's inequality for the martingale $Z-\widetilde{Z}$, along with Lemma 1, (7), (9) and (10), yields for any $t \in(0, T]$,

$$
\begin{aligned}
E \sup _{0 \leq s \leq t}\left|Z_{s \wedge S_{n}}-\widetilde{Z}_{s \wedge S_{n}}\right|^{4} & \leq c E\left|Z_{t \wedge S_{n}}-\widetilde{Z}_{t \wedge S_{n}}\right|^{4} \\
& \leq c T E \int_{0}^{t \wedge S_{n}}\left\|G\left(Y_{s}-X_{s}\right)-G\left(\widetilde{Y}_{s}-X_{s}\right)\right\|^{4} d s \\
& =c T E \int_{0}^{t \wedge S_{n}}\left\|H\left(\frac{V_{s}}{\left|V_{s}\right|}\right)-H\left(\frac{\widetilde{V}_{s}}{\left|\widetilde{V}_{s}\right|}\right)\right\|^{4} d s \\
& \leq c T E \int_{0}^{t \wedge S_{n}}\left|\frac{V_{s}}{\left|V_{s}\right|}-\frac{\widetilde{V}_{s}}{\left|\widetilde{V}_{s}\right|}\right|^{4} d s \\
& \leq c T n^{4} E \int_{0}^{t \wedge S_{n}}\left|Y_{s}-\widetilde{Y}_{s}\right|^{4} d s \\
& \leq c T n^{4} \int_{0}^{t} E \sup _{0 \leq u \leq s}\left|Y_{u \wedge S_{n}}-\widetilde{Y}_{u \wedge S_{n}}\right|^{4} d s \\
& \leq c T^{2} n^{4} \int_{0}^{t} E \sup _{0 \leq u \leq s}\left|Z_{u \wedge S_{n}}-\widetilde{Z}_{u \wedge S_{n}}\right|^{4} d s .
\end{aligned}
$$

Gronwall's inequality now shows that $E \sup _{0 \leq s \leq T}\left|Z_{s \wedge S_{n}}-\widetilde{Z}_{s \wedge S_{n}}\right|^{4}=0$. Hence $P\left(Z_{s}=\widetilde{Z}_{s}, 0 \leq s \leq S_{n}\right)=1$, for all $n$, and therefore $P\left(Z_{s}=\widetilde{Z}_{s}, 0 \leq s<\zeta \wedge \widetilde{\zeta}\right)=1$. This shows that $Z$ and $\widetilde{Z}$ are indistinguishable on $[0, T]$. Since $T$ is arbitrary, the same is true on $[0, \infty)$. This completes the proof of pathwise uniqueness. 
Existence of strong solutions. By (77), the restriction of the matrix-valued mapping $x \mapsto G(x)$ to $\mathbb{R}^{d} \backslash B_{\varepsilon}(0)$ is globally Lipschitz with respect to $\|\cdot\|$. Therefore, for every $n \in \mathbb{N}$, one can find a globally Lipschitz function $G^{n}$ on $\mathbb{R}^{d}$ that agrees with $G$ outside $B_{1 / n}(0)$. Let $\lambda_{n}<\infty$ denote the Lipschitz constant of $G^{n}$. We are given a probability space $(\Omega, F, P)$ with a Brownian motion $W$ and the filtration $\left(F_{t}^{W}\right)$ generated by $W$. Denote $X=\Gamma(x+W)$. We will first show that for any $n$ there exists a continuous adapted process $Z^{n}$ such that

$$
Z_{t}^{n}=\int_{0}^{t} G^{n}\left(\Gamma\left(y+Z^{n}\right)_{s}-X_{s}\right) d W_{s}
$$

But before that, let us note that pathwise uniqueness holds for (11). The proof is similar to that of pathwise uniqueness for (8) but simpler, so it is omitted.

In this paragraph we drop the dependence on $n$ in the notation of the processes involved. Recall the space $\mathbf{Z}$ defined before the statement of Lemma 1 . Let $F$ map $Z \in \mathbf{Z}$ to

$$
F(Z)_{t}=\int_{0}^{t} G^{n}\left(\Gamma(y+Z)_{s}-X_{s}\right) d W_{s}
$$

Suppose $Z, Z^{\prime} \in \mathbf{Z}$. By Lemma1 Doob's inequality for the martingale $F(Z)-F\left(Z^{\prime}\right)$ and (10), we have for $t \in(0, T]$,

$$
\begin{aligned}
E \sup _{s \leq t} & \left|F(Z)_{s}-F\left(Z^{\prime}\right)_{s}\right|^{4} \leq c E\left|F(Z)_{t}-F\left(Z^{\prime}\right)_{t}\right|^{4} \\
& \leq c t E \int_{0}^{t}\left\|G^{n}\left(\Gamma(y+Z)_{s}-X_{s}\right)-G^{n}\left(\Gamma\left(y+Z^{\prime}\right)_{s}-X_{s}\right)\right\|^{4} d s \\
& \leq c \lambda_{n}^{4} t E \int_{0}^{t}\left|\Gamma(y+Z)_{s}-\Gamma\left(y+Z^{\prime}\right)_{s}\right|^{4} d s \\
& \leq c \lambda_{n}^{4} T^{2} \int_{0}^{t} E \sup _{0 \leq u \leq s}\left|Z_{u}-Z_{u}^{\prime}\right|^{4} d s .
\end{aligned}
$$

Let $Z^{(0)}=\mathbf{0}$, and for $k \in \mathbb{N}$ let $Z^{(k)}=F\left(Z^{(k-1)}\right)$. Since $G^{n}$ is bounded, there is $c$ such that $\left\|Z^{(1)}-Z^{(0)}\right\|_{T} \leq c$. Iterating (12), it easily follows that

$$
\left\|Z^{(k+1)}-Z^{(k)}\right\|_{T}^{4} \leq c \frac{c_{1}^{k}}{k !}
$$

where $c_{1}$ is a constant that may depend on $n$ and $T$. Hence by Chebyshev's inequality,

$$
P\left(\sup _{s \leq T}\left|Z_{s}^{(k+1)}-Z_{s}^{(k)}\right| \geq 2^{-k}\right) \leq c\left(16 c_{1}\right)^{k} / k !,
$$

and the Borel-Cantelli lemma shows that with probability one,

$$
\sup _{0 \leq s \leq T}\left|Z_{s}^{(k+1)}-Z_{s}^{(k)}\right| \leq 2^{-k}
$$

for all sufficiently large $k$. Hence the paths of $Z^{(k)}$ converge in the uniform topology, a.s., say, to $Z$. Again using (12), we have $\left\|F\left(Z^{(k)}\right)-F(Z)\right\|_{T}^{4} \leq c\left\|Z^{(k)}-Z\right\|_{T}^{4}$, and as a result $F\left(Z^{(k)}\right)$ converge a.s. to $F(Z)$. We see that $Z$ is a fixed point of $F$. Since $T$ is arbitrary, we conclude that there exists a continuous, $\left(F_{t}^{W}\right)$-adapted process satisfying (11) for all $t \in[0, \infty)$ a.s.

We reintroduce the dependence on $n$ in our notation. Let $Z^{n}$ denote the process in (11) and set $Y^{n}=\Gamma\left(y+Z^{n}\right)$. Let $\tau_{n}=\inf \left\{s:\left|Y_{s}^{n}-X_{s}\right| \leq 1 / n\right\}$. Since we have 
uniqueness for (11), it is clear that on $\left[0, \tau_{n}\right]$, the processes $Z^{n}$ and $Z^{n+1}$ agree a.s. Setting $\zeta=\lim _{n} \tau_{n}$ and defining

$$
Z_{t}=1_{t<\zeta} \lim _{n \rightarrow \infty} Z^{n}(t)+1_{t \geq \zeta}\left(W_{t}-W_{\zeta}\right),
$$

we obtain a continuous and adapted process $Z$. We see that $(8)$ is satisfied for all $t \in[0, \infty)$ a.s. Therefore there exists a strong solution relative to $W$.

Strong Markov property. Let $\left(\Omega, F,\left(F_{t}\right), P, W\right)$ be a complete filtered probability space and a Brownian motion. Let $U=(X, Y)$ denote the unique solution to (44), (5) and (8). For $u=(x, y) \in(\bar{D})^{2}$, let $P^{u}$ denote the measure induced by $U$ on $\left(C\left([0, \infty): \mathbb{R}^{2 d}\right), \mathcal{B}\left(C\left([0, \infty): \mathbb{R}^{2 d}\right)\right)\right)$, assuming $U_{0}=u$. Let $E^{u}$ denote the expectation with respect to $P^{u}$. The transition function for the process $U$ is defined as

$$
P_{t}(u, f)=E^{u}\left(f\left(U_{t}\right)\right)
$$

for all $u \in(\bar{D})^{2}$ and bounded Borel measurable functions $f$. Given the results on existence and uniqueness, and the properties of the Skorohod map quoted before, we have the following.

Corollary 2. The process $U$ is strong Markov, i.e., for any a.s. finite $\left(F_{t}\right)$-stopping time $T$ and bounded Borel function $f$, one has $P$-a.s., for all $s \geq 0$,

$$
E\left[f\left(U_{T+s}\right) \mid F_{T}\right]=P_{s}\left(U_{T}, f\right) .
$$

Proof. We take here the approach of [21], Theorem V.32. For each finite stopping time $T$, note that $\widetilde{W}_{s}=W_{T+s}-W_{T}, s \geq 0$, is a Brownian motion (cf., e.g., [21], Theorem I.32), and consider the unique solution $\widetilde{Z}$ to the following equation, with $u=(x, y) \in(\bar{D})^{2}$,

$$
\widetilde{Z}_{t}=\int_{0}^{t \wedge \widetilde{\zeta}} G\left(\Gamma(y+\widetilde{Z})_{s}-\Gamma(x+\widetilde{W})_{s}\right) d \widetilde{W}_{s}+1_{t \geq \widetilde{\zeta}}\left(\widetilde{W}_{t}-\widetilde{W}_{\zeta}\right),
$$

where $\widetilde{\zeta}=\inf \left\{s: \Gamma(y+\widetilde{Z})_{s}=\Gamma(x+\widetilde{W})_{s}\right\}$. To take into account the dependence on the stopping time and the initial condition, we denote $\widetilde{Z}_{t}$ above by $Z(u, T, t)$, and we also let

$$
U(u, T, t)=\left(\Gamma\left(x+W_{\cdot+T}\right)_{t}, \Gamma(y+Z(u, T, \cdot))_{t}\right) .
$$

Denote the Borel sigma-field of subsets of $(\bar{D})^{2}$ [resp., $\left.\mathbb{R}_{+}\right]$by $\mathcal{U}$ [resp., $\left.\mathcal{R}_{+}\right]$. We will show that, for a given stopping time, the mapping $(u, t, \omega) \mapsto Z(u, T, t)$ has a $\mathcal{U} \otimes \mathcal{R}_{+} \otimes \mathcal{F}$ jointly measurable version, and hence so does $U(u, T, t)$. We will use these versions in the rest of the proof. Recall the processes $Z^{(k)}$ and $Z^{n}$ of the proof of Theorem 2. Recall also that for each $n, Z^{(k)}$ converge uniformly on compacts in probability to $Z^{n}$. Since each $Z^{(k)}$ is jointly measurable, this implies that $Z^{n}$ is also (cf. 21). Since $Z$ is the pointwise limit of $Z^{n}$ as $n \rightarrow \infty$, this shows that $Z$ is jointly measurable. The same argument applies to $Z(u, T, t)$ for any fixed stopping time $T$. A similar measurability property holds for $U(u, T, t)$ by the uniform continuity of $\Gamma$ on compact sets.

Fix a finite stopping time $T$ and set $F^{*}=\sigma\left\{W_{T+u}-W_{T}: u \geq 0\right\}$. Then $F^{*}$ is independent of $F_{T}$ under $P$, since $W$ is an $\left(F_{t}\right)$-Brownian motion. Note that $Z(u, T, t)$ is measurable with respect to $F^{*}$. By the uniqueness results for the Skorohod map (the uniqueness holds in the deterministic sense), $\Gamma(R)_{T+s}=$ $\Gamma\left(\Gamma(R)_{T}-R_{T}+R_{T+} .\right)_{s}, s \geq 0$, whenever $R$ is an $\left(F_{t}\right)$-Brownian motion. Fix a $u$ 
for the moment and denote $Z_{t}=Z(u, 0, t)$. Then we have for $t \geq 0$, on the event $\{\zeta>T+t\}$,

$$
\begin{aligned}
Z_{T+t}-Z_{T} & =\int_{T}^{T+t} G\left(\Gamma(y+Z)_{s}-\Gamma(x+W)_{s}\right) d W_{s} \\
(14) & =\int_{0}^{t} G\left(\Gamma\left(\Gamma(y+Z)_{T}+\left(Z_{T+\cdot}-Z_{T}\right)\right)_{s}-\Gamma\left(\Gamma(x+W)_{T}+\widetilde{W}\right)_{s}\right) d \widetilde{W}_{s} .
\end{aligned}
$$

Let $\bar{\zeta}=\inf \left\{s \geq 0: \Gamma\left(y+Z_{T+} \cdot\right)_{s}=\Gamma\left(x+W_{T+}\right)_{s}\right\}$, and note that $\bar{\zeta}=0$ on $\zeta \leq T$. On the event $\{\zeta \leq T+t\}=\{\bar{\zeta} \leq t\}$, we have,

$$
\begin{aligned}
Z_{T+t}-Z_{T} & =Z_{\zeta}+W_{T+t}-W_{\zeta} \\
& =Z_{\bar{\zeta}}+\widetilde{W}_{t}-\widetilde{W}_{\bar{\zeta}} .
\end{aligned}
$$

Equations (14) and (15) show that $Z_{T+t}-Z_{T}$ is a solution to (13) with the initial condition $U(u, 0, T)$. By uniqueness of solutions we therefore have

$$
Z_{T+t}-Z_{T} \equiv Z(u, 0, T+t)-Z(u, 0, T)=Z(U(u, 0, T), T, t) .
$$

It follows that $P$-a.s., for all $t \geq 0$,

$$
U(u, 0, T+t)=U(U(u, 0, T), T, t) .
$$

We will use the following standard fact. If $\Phi(h, \cdot)$ is independent of the $\sigma$-field $\mathcal{H}$ for every $h$ and $H$ is $\mathcal{H}$-measurable, then $E(\Phi(H, \cdot) \mid \mathcal{H})=\phi(H)$ a.s., where $\phi(h)=E(\Phi(h, \cdot))$. Hence, for any bounded Borel $f$,

$$
\begin{aligned}
E\left\{f(U(u, 0, T+t)) \mid F_{T}\right\} & =E\left\{f(U(U(u, 0, T), T, t)) \mid F_{T}\right\} \\
& =g(U(u, 0, T)),
\end{aligned}
$$

where $g(r)=E f(U(r, T, t))$. However, by pathwise uniqueness of solutions to (13), under $P, Z(u, T, \cdot)$ and $Z(u, 0, \cdot)$ are equal in law, and therefore so are $U(u, T, \cdot)$ and $U(u, 0, \cdot)$. Consequently, $g(r)=E f(U(r, 0, t))=P_{t}(r, f)$. This shows that

$$
E\left\{f(U(u, 0, T+t)) \mid F_{T}\right\}=P_{t}(U(u, 0, T), f) .
$$

Remark. Fix some $u$, consider the probability space $\left(\Omega, F, P^{u}\right)$, where $P^{u}$ is the measure induced by $U$ when started at $u$, and let $\left(\widehat{F}_{t}\right)$ be the filtration generated by $U$. Then by Corollary 2 for any a.s. finite $\left(\widehat{F}_{t}\right)$-stopping time $T$, the random variable $E\left[f\left(U_{T+s}\right) \mid F_{T}\right]=P_{s}\left(U_{T}, f\right)$ is measurable with respect to $\sigma\left(U_{T}\right)$. It follows that it is measurable with respect to $\widehat{F}_{T}$, and therefore $P^{u}$-a.s.,

$$
E^{u}\left[f\left(U_{T+s}\right) \mid \widehat{F}_{T}\right]=E\left[f\left(U_{T+s}\right) \mid F_{T}\right]=P_{s}\left(U_{T}, f\right) .
$$

Next we will show that the "mirror coupling" $(X, Y)$ has in fact the mirror property.

Proposition 1. Let $(X, Y)$ be a mirror coupling of reflecting Brownian motions, let $A$ be an event in $F$, and let $\sigma, \tau$ be two a.s. finite $F$-measurable times with $\sigma \leq \tau$ a.s. on $A$, such that $X_{s}, Y_{s} \notin \partial D$ and $X_{s} \neq Y_{s}$ for all $s \in[\sigma, \tau)$ a.s. on $A$. Then a.s. on $A$,

$$
\left(X_{s}+Y_{s}\right) \cdot\left(Y_{\sigma}-X_{\sigma}\right)=\left(X_{\sigma}+Y_{\sigma}\right) \cdot\left(Y_{\sigma}-X_{\sigma}\right), \quad \text { for all } s \in[\sigma, \tau] .
$$


Proof. Recall that in our notation, $d X=d W+d L, d Y=d Z+d M, L$ and $M$ are the boundary terms for $X$ and $Y$, and $d Z=H(m) d W=d W-2 m m \cdot d W$. Here $m_{s}=V_{s} /\left|V_{s}\right|$ on $\{s<\zeta\}$, where $V_{s}=Y_{s}-X_{s}$. Applying Itô's formula, one finds that

$$
d m_{s}=\left|V_{s}\right|^{-1}\left(d M_{s}-d L_{s}\right)-\left|V_{s}\right|^{-1} m_{s} m_{s} \cdot\left(d M_{s}-d L_{s}\right) .
$$

This shows that the mirror does not move within $[\sigma, \tau]$ on $A$. Hence for $s \in[\sigma, \tau]$,

$$
Z_{s}=Z_{\sigma}+G\left(Y_{\sigma}-X_{\sigma}\right)\left(W_{s}-W_{\sigma}\right) .
$$

Since for $s \in[\sigma, \tau]$,

$$
X_{s}=X_{\sigma}+W_{s}-W_{\sigma}, \quad Y_{s}=Y_{\sigma}+Z_{s}-Z_{\sigma},
$$

(16) follows.

Let $\left(e_{1}, e_{2}\right)$ be the usual orthonormal basis for $\mathbb{R}^{2}$ and let

$$
e_{1}^{\prime}=\left(e_{1}-e_{2}\right) / \sqrt{2}, \quad e_{2}^{\prime}=\left(e_{1}+e_{2}\right) / \sqrt{2} .
$$

For $x, y \in \mathbb{R}^{2}$, let $x \leq y$ mean

$$
x \cdot e_{i}^{\prime} \leq y \cdot e_{i}^{\prime}, \quad i=1,2 .
$$

The following "order preserving" property of mirror couplings in lip domains is one of the crucial ingredients of the main argument.

Proposition 2. Suppose $D \subset \mathbb{R}^{2}$ is a piecewise $C^{2}$-smooth lip domain for which the defining functions $f_{1}, f_{2}$ (cf. equation (1D) are both Lipschitz with constants strictly less than 1. Let $(X, Y)$ be a mirror coupling of reflecting Brownian motions in $D$, starting from $(x, y) \in(\bar{D})^{2}$. If $x \leq y$, then $X_{t} \leq Y_{t}$ for all $t \geq 0$, a.s.

Proof. Recall the definition of $m_{s}$ from the proof of Proposition 1 Let $a_{s}$ be the unit vector perpendicular to $m_{s}$ such that, in complex number notation, $i a_{s}=m_{s}$. Then the identity (17) is equivalent to

$$
d m_{s}=\left|V_{s}\right|^{-1} a_{s} a_{s} \cdot\left(d M_{s}-d L_{s}\right) .
$$

Let $\tau=\inf \left\{t: X_{t} \not \leq Y_{t}\right\}$ and $\Omega_{1}=\{\tau<\infty\}$. We will show that $\Omega_{1}$ has probability 0 . Suppose $\tau<\infty$. Since a normally reflecting Brownian motion does not hit a fixed point on the boundary, we can assume that $X_{\tau}$ and $Y_{\tau}$ are not at any of the vertices of $\partial D$. Let $\partial_{+} D\left[\partial_{-} D\right]$ denote the intersection of the boundary $\partial D$ with the graph of $f_{2}$ [respectively, $f_{1}$ ]. Because the Lipschitz constants of $f_{1}$ and $f_{2}$ are assumed to be less than 1, it cannot happen that both $X_{\tau}$ and $Y_{\tau}$ are on the same side $\partial_{+} D$ or $\partial_{-} D$ of the boundary. Assume without loss of generality that $Y_{\tau} \in \partial_{+} D$. Then $X_{\tau} \in D \cup \partial_{-} D, m_{\tau}=e_{2}^{\prime}$ and $a_{\tau}=e_{1}^{\prime}$. It follows from the definition of $\tau$ that for every $\varepsilon>0$ there exists $t \in(\tau, \tau+\varepsilon)$ with $m_{t} \cdot e_{1}^{\prime}<0$. Since $a_{t}$ is continuous, we can find small $\varepsilon_{0}>0$ such that for $s \in\left(\tau, \tau+\varepsilon_{0}\right]$, (i) $a_{s} \cdot e_{1}^{\prime} \geq 0$; (ii) $a_{s} \cdot \nu\left(Y_{s}\right) \geq 0$ if $Y_{s} \in \partial D$; and (iii) $a_{s} \cdot \nu\left(X_{s}\right) \leq 0$ if $X_{s} \in \partial D$. In (ii) and (iii) we have used the fact that $Y_{\tau} \in \partial_{+} D$ and $X_{\tau} \notin \partial_{+} D$ and the assumption that the Lipschitz constants of $f_{k}$ 's are less than 1 . For all $t \in\left(\tau, \tau+\varepsilon_{0}\right]$,

$$
\begin{aligned}
m_{t} \cdot e_{1}^{\prime} & =m_{\tau} \cdot e_{1}^{\prime}+\int_{\tau}^{t}\left|V_{s}\right|^{-1} a_{s} \cdot e_{1}^{\prime} a_{s} \cdot\left(d M_{s}-d L_{s}\right) \\
& =\int_{\tau}^{t}\left|V_{s}\right|^{-1} a_{s} \cdot e_{1}^{\prime} a_{s} \cdot \nu\left(Y_{s}\right) d|M|_{s}-\int_{\tau}^{t}\left|V_{s}\right|^{-1} a_{s} \cdot e_{1}^{\prime} a_{s} \cdot \nu\left(X_{s}\right) d|L|_{s}
\end{aligned}
$$


The first integral in the last line is nonnegative and the second one is nonpositive. This contradicts the fact that $m_{t} \cdot e_{1}^{\prime}<0$ for some $t \in\left(\tau, \tau+\varepsilon_{0}\right)$ and so it shows that $\Omega_{1}$ has probability zero.

\section{Conditioned MirRor COUPLings}

Throughout this section, we fix a lip domain $D \subset \mathbb{R}^{2}$ and a sequence of lip domains $D^{n}$ increasing to $D$, such that the defining functions $f_{1}^{n}, f_{2}^{n}$ (cf. equation (11)) are $C^{2}$-smooth and Lipschitz with the Lipschitz constants strictly less than 1 . Recall that a lip domain is a Lipschitz domain, i.e., its boundary can be represented as the graph of a Lipschitz function with constant $\lambda$ in some neighborhood of every boundary point (the point here is that the extreme left and right points have to be included). We will assume without loss of generality that there exist constants $\lambda<\infty$ and $r_{0}>0$, not depending on $n$, such that for every $n$ and $x \in \partial D^{n}$, the set $\partial D^{n} \cap B_{r_{0}}(x)$ can be represented as the graph of a (single) function with Lipschitz constant $\lambda$. Moreover, the same applies to any $x \in \partial D$ and $\partial D \cap B_{r_{0}}(x)$. For each $D^{n}$, Corollary 2 and Propositions 1 and 2 apply.

Let $D_{\varepsilon}=\{x \in D: \operatorname{dist}(x, \partial D) \geq \varepsilon\}$ and $D_{\varepsilon}^{n}=\left\{x \in D^{n}: \operatorname{dist}\left(x, \partial D^{n}\right) \geq \varepsilon\right\}$. The hitting time of a set $A$ by a process $W$ will be denoted $T^{W}(A)$, or simply $T(A)$, if no confusion may arise.

Lemma 2. Let $Q_{x, y}^{n}$ denote any probability measure under which $(X, Y)$ is a strong Markov process, and $X$ [respectively, $Y$ ] is a reflected Brownian motion in $D^{n}$ starting from $x[y]$. Then there are constants $\delta, \sigma>0$, such that for any $\rho>0, n$, $x, y \in \overline{D^{n}},|x-y| \geq \rho$,

where

$$
Q_{x, y}^{n}\left(T_{0} \leq \sigma \rho^{2}\right) \geq 1 / 2,
$$

$$
T_{0}=\inf \left\{t>0: X_{t}, Y_{t} \in D_{\delta \rho}^{n}, X_{t} \in B_{\rho / 16}(x), Y_{t} \in B_{\rho / 16}(y)\right\} .
$$

Proof. The lemma will be proved in two steps.

Step 1. In this step, we will show that there exist $\gamma, \varepsilon_{0}>0$, such that for any $\varepsilon \in\left(0, \varepsilon_{0}\right), n$, and $x \in \overline{D^{n}}$,

$$
Q_{x, y}^{n}\left(T^{X}\left(D_{\varepsilon} \cap B_{\gamma \varepsilon}(x)\right)<\varepsilon^{2}\right)>9 / 10 .
$$

Recall the Lipschitz constant $\lambda$ defined before the lemma and suppose that $f$ : $\mathbb{R} \rightarrow \mathbb{R}$ is Lipschitz with constant $\lambda$, and $f(0)=0$. Let $A=\left\{x \in \mathbb{R}^{2}: x_{2}>f\left(x_{1}\right)\right\}$,

$$
\begin{aligned}
\Delta(x, r, h) & =\left\{y \in A:\left|y_{1}-x_{1}\right|<r, y_{2}<f\left(y_{1}\right)+h\right\}, \\
\partial_{u} \Delta(x, r, h) & =\left\{y \in \partial \Delta(x, r, h): y_{2}=f\left(y_{1}\right)+h\right\}, \\
\partial_{s} \Delta(x, r, h) & =\left\{y \in \partial \Delta(x, r, h):\left|y_{1}-x_{1}\right|=r\right\} .
\end{aligned}
$$

Let $P_{x}^{n}$ be the distribution of the reflected Brownian motion in $A$ starting from $x$. The function

$$
g(y)=P_{y}^{n}\left(T^{X}\left(\partial_{u} \Delta((0,0), 1,1)\right) \leq T^{X}\left(\partial_{s} \Delta((0,0), 1,1)\right)\right)
$$

is harmonic in $\Delta((0,0), 1,1)$. By a Harnack inequality proved for such functions in 6], $g(y) \geq c g((0,1 / 2))$ for some $c$ depending only on $\lambda$ and all $y \in \Delta((0,0), 1 / 2,1)$. Hence, for some $p>0$ depending only on $\lambda$, the reflected Brownian motion starting from any point of $\Delta((0,0), 1 / 2,1)$ exits $\Delta((0,0), 1,1)$ through $\partial_{u} \Delta((0,0), 1,1)$ with probability greater than $p$. By the strong Markov property applied at the hitting time of $\partial \Delta((0,0), 1,1) \backslash \partial A$, the process starting from any point in $\Delta((0,0), 1 / 2,1)$ 
exits $\Delta((0,0), 2,1)$ through $\partial_{u} \Delta((0,0), 2,1)$ with probability greater than 1 $(1-p)^{2}$. By induction, the process starting from a point of $\Delta((0,0), 1 / 2,1)$ exits $\Delta((0,0), \gamma, 1)$ through $\partial_{u} \Delta((0,0), \gamma, 1)$ with probability greater than $1-(1-p)^{\gamma}$, for integer $\gamma>1$. Fix a $\gamma$ so large that $1-(1-p)^{\gamma}>1-1 / 20$.

By Theorem 2.3 of [6], $P_{x}^{n}\left(X_{1} \in d y\right) \leq c_{1} \exp \left(c_{2}|x-y|^{2}\right) d y$, where the constants $c_{1}$ and $c_{2}$ depend only on $\lambda$. Choose $h>0$ so small that $P_{x}^{n}\left(X_{1} \in \Delta((0,0), \gamma, h)\right)<$ $1 / 20$ for $x \in \Delta((0,0), 1 / 2, h)$. We see that the probability that the process starting from $\Delta((0,0), 1 / 2, h)$ is still in $\Delta((0,0), \gamma, h)$ at time 1 or exited it through $\partial_{s} \Delta((0,0), \gamma, h)$ by that time is bounded by $1 / 20+1 / 20=1 / 10$. Now (18) follows by scaling.

Step 2. Consider small $\bar{\delta}>0$, large $\bar{t}<\infty$, and finite stopping times

$T_{1}^{\prime}=T^{X}\left(D_{\bar{\delta} \rho}^{n} \cap B_{\gamma \bar{\delta} \rho}(x)\right) \wedge \bar{t}, \quad T_{1}^{\prime \prime}=T^{Y}\left(D_{\bar{\delta} \rho}^{n} \cap B_{\gamma \bar{\delta} \rho}(y)\right) \wedge \bar{t}, \quad T_{1}=T_{1}^{\prime} \wedge T_{1}^{\prime \prime}$.

Using Step 1 with $\varepsilon=\bar{\delta} \rho$, one has for large $\bar{t}$,

$$
Q_{x, y}^{n}\left(T_{1}^{\prime}<(\bar{\delta} \rho)^{2}\right)>9 / 10
$$

and, similarly, $Q_{x, y}^{n}\left(T_{1}^{\prime \prime}<(\bar{\delta} \rho)^{2}\right)>9 / 10$. Let

$$
C_{\bar{\delta}, \eta}=\left\{X_{t} \in B_{\bar{\delta} \rho / 2}\left(X_{T_{1}}\right), t \in\left[T_{1}, T_{1}+\eta^{2} \bar{\delta}^{2} \rho^{2} / 4\right]\right\} .
$$

If $T_{1}^{\prime} \leq T_{1}^{\prime \prime}$, then $B_{\bar{\delta} \rho / 2}\left(X_{T_{1}}\right) \subset D^{n}$ and by choosing $\eta \in(0,1)$ small, one can have

$$
Q_{x, y}^{n}\left(C_{\bar{\delta}, \eta} \mid \mathcal{F}_{T_{1}}, T_{1}^{\prime} \leq T_{1}^{\prime \prime}\right) \geq 9 / 10 \text {. }
$$

Let $T_{2}=\inf \left\{t>T_{1}: Y_{t} \in D_{\eta \bar{\delta} \rho / 2} \cap B_{\gamma \eta \bar{\delta} \rho / 2}\left(Y_{T_{1}}\right)\right\}$. Using Step 1 with $\varepsilon=\eta \bar{\delta} \rho / 2$ and the strong Markov property,

$$
Q_{x, y}^{n}\left(T_{2}<T_{1}+(\eta \bar{\delta} \rho)^{2} / 4 \mid \mathcal{F}_{T_{1}}, T_{1}^{\prime} \leq T_{1}^{\prime \prime}\right)>9 / 10 .
$$

Hence,

$$
Q_{x, y}^{n}\left(C_{\bar{\delta}, \eta}, T_{2}<T_{1}+(\eta \bar{\delta} \rho)^{2} / 4 \mid \mathcal{F}_{T_{1}}, T_{1}^{\prime} \leq T_{1}^{\prime \prime}\right)>8 / 10 .
$$

A similar formula holds in the case $T_{1}^{\prime \prime} \leq T_{1}^{\prime}$. Combining this with (19), we obtain

$$
Q_{x, y}^{n}\left(T_{0} \leq(\bar{\delta} \rho)^{2}+(\eta \bar{\delta} \rho)^{2} / 4\right) \geq(9 / 10)(8 / 10)>1 / 2 .
$$

Now make $\bar{\delta}>0$ smaller, if necessary, so that $(\gamma \bar{\delta} \rho+\bar{\delta} \rho / 2) \vee(\gamma \bar{\delta} \rho+\gamma \eta \bar{\delta} \rho / 2)<\rho / 16$, and thus the lemma holds with $\delta=\eta \bar{\delta} / 2$.

In the rest of this section, we denote by $P_{x, y}^{n}$ a measure under which $(X, Y)$ is a mirror coupling of reflected Brownian motions in $D^{n}$. Let $\tau$ be the coupling time for $X$ and $Y$. Following [9], we let $\mathcal{D}^{n}=\left\{(x, y) \in \overline{D^{n}} \times \overline{D^{n}}: x \neq y\right\}$, $\mathcal{D}^{n}(\varepsilon)=\left\{(x, y) \in \overline{D^{n}} \times \overline{D^{n}}:|x-y| \geq \varepsilon\right\}$, and $\widehat{\mathcal{D}}^{n}(\varepsilon)=\mathcal{D}^{n} \backslash \mathcal{D}^{n}(\varepsilon)$.

Lemma 3. (i) There are constants $\alpha, c, a>0$ such that for all $\varepsilon \in(0, a)$ and all $(x, y) \in \mathcal{D}^{n}(\varepsilon)$,

$$
P_{x, y}^{n}\left(T\left(\mathcal{D}^{n}(a)\right)<\tau\right) \geq c \varepsilon^{\alpha} .
$$

(ii) There is a constant $c<\infty$ such that for all $\varepsilon>0$ and $(x, y) \in \widehat{\mathcal{D}}^{n}(\varepsilon)$,

$$
E_{x, y}^{n} T\left(\left(\widehat{\mathcal{D}}^{n}(\varepsilon)\right)^{c}\right) \leq c \varepsilon^{2} .
$$

(iii) For any $u>0$ we can find $\varepsilon_{0}, c>0$ such that $\mathcal{D}^{1}\left(2 \varepsilon_{0}\right)$ has a nonempty interior and

$$
P_{x, y}^{n}\left(\tau>t,\left(X_{t}, Y_{t}\right) \in \mathcal{D}^{n}\left(\varepsilon_{0}\right)\right) \geq c,
$$

for all $n,(x, y) \in \mathcal{D}^{n}\left(2 \varepsilon_{0}\right)$, and $t \in[u / 4, u]$. 
Proof. To keep the notation simple, the superscript $n$ will be dropped from $D^{n}, D_{\varepsilon}^{n}$, $P_{x, y}^{n}, \mathcal{D}^{n}, \mathcal{D}^{n}(\varepsilon)$ and $\widehat{\mathcal{D}}^{n}(\varepsilon)$ throughout this proof. All constants in all estimates will be tacitly assumed to be independent of $n$.

Let us outline the strategy of the proof. The main step in the proof of (i) will be to show that starting at any $x, y \in \bar{D}, x \leq y,|x-y|=\rho>0$, the probability of hitting $\mathcal{D}(2 \rho)$ before exiting $\mathcal{D}$ is bounded below by some $p>0$, independent of $\rho$ and $n$. The main estimate in the proof of (ii) will be that the probability of exiting $\mathcal{D}$ before time $c \rho^{2}$ is bounded below by some $p>0$, where $c$ and $p$ are independent of $\rho$ and $n$. These two estimates will be obtained by identifying events $H, K$ and a time $S$, with the properties

$$
H \subset\left\{S<\tau,\left|X_{S}-Y_{S}\right| \geq 2 \rho\right\},
$$

while

$$
P_{x, y}(H) \geq p
$$

and

$$
K \subset\left\{\tau \leq c \rho^{2}\right\}, \quad P_{x, y}(K) \geq p .
$$

Recall the notation $x^{*}$ and $y^{*}$ for the unique points in $\bar{D}$ for which $x^{*} \leq x \leq y^{*}$, all $x \in D$. See the beginning of this section for the definition of the Lipschitz constant $\lambda$ characterizing $D$. Let $\partial^{+} D$ [respectively, $\partial^{-} D$ ] denote the upper [lower] part of the boundary $\partial D$. Let $a>0$ be so small that (I) whenever $B_{32 a}(x)$ intersects both $\partial^{+} D$ and $\partial^{-} D$, one has $\left|x-x^{*}\right| \wedge\left|x-y^{*}\right| \leq 64 \lambda a$; (II) there is a coordinate system in which $D \cap B_{128 \lambda a}\left(x^{*}\right)$ is equal to the set of points above the graph of a Lipschitz function with constant $\lambda$; and (III) a similar statement holds for $y^{*}$. We will consider $x, y \in \bar{D}$ with $|x-y|=\rho$ and $\rho \in(0, a)$. There are three cases: (a) $B_{32 \rho}(x)$ intersects either $\partial^{+} D$ or $\partial^{-} D$, but not both, (b) $B_{32 \rho}(x)$ intersects both parts of the boundary, (c) $B_{32 \rho}(x)$ is contained in $D$. In case (c), the existence of $H, K, S$ for which (20), (21) and (22) are valid follows from a simple scaling argument for the planar Brownian motion, and we therefore omit the details.

(a) From now on, we will refer to $X$ and $Y$ as "Brownian particles" or simply "particles." In case (a) there are two possibilities according to whether the particles $X$ and $Y$ are close to the upper or lower boundary. We consider only the lower boundary, since the other case can be treated similarly.

Recall that $\left(e_{1}, e_{2}\right)$ is the usual coordinate system in $\mathbb{R}^{2}$. Within case (a) we consider three subcases: (a1) the angle $\theta$, which $y-x$ forms with $e_{1}$, is within $[-\pi / 4,-\pi / 16] ;$ (a2) $\theta \in[\pi / 16, \pi / 4]$; and (a3) $\theta \in(-\pi / 16, \pi / 16)$.

(a1) Assume $x$ and $y$ are as in case (a1). Let $\sigma, \delta>0$ and let $T_{0}$ be as in Lemma 2 and let $H_{0}$ denote the event $\left\{T_{0} \leq \sigma \rho^{2}\right\}$. By definition, on $H_{0}, X_{T_{0}}, Y_{T_{0}} \in D_{\delta \rho}$, and $X_{T_{0}} \in B_{\rho / 16}(x), Y_{T_{0}} \in B_{\rho / 16}(y)$. A simple calculation, using the fact that $\theta \in[-\pi / 4,-\pi / 16]$, shows that $X_{T_{0}} \cdot e_{2} \geq Y_{T_{0}} \cdot e_{2}$. Define

$$
A^{X}(x, s, v, \gamma, T)=\left\{\left|X_{t}-x-v t\right|<\gamma, t \in[s, T]\right\} .
$$

If $H_{0}$ holds, then $Y_{T_{0}}-X_{T_{0}} \neq 0$ and we can define orthogonal vectors $\left(\xi_{1}, \xi_{2}\right)$ as $\xi_{1}=\left(Y_{T_{0}}-X_{T_{0}}\right) /\left|Y_{T_{0}}-X_{T_{0}}\right|$ and $\xi_{2}$ is the unit vector orthogonal to $\xi_{1}$ for which $\xi_{2} \cdot e_{2}>0$. We will define an event $H_{1} \subset H_{0}$ which represents, intuitively speaking, a motion of $X$ along a tube that starts from $X_{T_{0}}$ and is parallel to the mirror. Namely,

$$
H_{1}=H_{0} \cap A^{X}\left(X_{T_{0}}, T_{0}, \rho^{-1} \xi_{2}, h \rho, T_{1}\right),
$$


where $T_{1}=T_{0}+8 \rho^{2}$, and $h=(\delta / 2) \wedge(1 / 16)$. Note that on $H_{1}$, neither $X$ nor $Y$ hits the boundary $\partial D$ within the time interval $\left[T_{0}, T_{1}\right]$ - this follows easily from the fact that $B_{h \rho}\left(X_{T_{0}}\right)$ and $B_{h \rho}\left(Y_{T_{0}}\right)$ are contained in $D$ and that, locally, $D$ is the set of points above the graph of a Lipschitz function with constant 1 . Also note that the fact that $X_{T_{0}} \cdot e_{2} \geq Y_{T_{0}} \cdot e_{2}$ on $H_{0}$ implies that $\xi_{2} \cdot e_{1} \geq 0$, which shows that, on $H_{1}$,

$$
X_{T_{1}} \cdot e_{1} \geq X_{T_{0}} \cdot e_{1}-h \rho .
$$

On $H_{1}$, the distance between the particles at time $T_{1}$ is within $[(1-1 / 8-2 h) \rho$, $(1+1 / 8+2 h) \rho]$. To make the distance larger, we consider an event $H$, on which $X$ moves within a tube starting from $X_{T_{1}}$ in the direction of $-e_{1}$. Specifically,

$$
H=H_{1} \cap A^{X}\left(X_{T_{1}}, T_{1},-\rho^{-1} e_{1}, h \rho, T_{2}\right),
$$

where $T_{2}=T_{1}+5 \rho^{2}$. Let $\Lambda \subset \mathbb{R}^{2}$ consist of two line segments - the first one starts from $X_{T_{0}}$ and goes $8 \rho$ units in the $\xi_{2}$ direction; the second one starts at the endpoint of the first one and goes $5 \rho$ units in the $-e_{1}$ direction. If $H_{0}$ holds, then the distance of $\Lambda$ from $\partial D$ is greater than $h \rho$, because the boundary is locally Lipschitz with constant 1 . Hence, if $H$ occurs, then $X$ does not hit the boundary within the time interval $\left[T_{0}, T_{2}\right]$.

Recall that the "mirror" is the line of symmetry between $X_{t}$ and $Y_{t}$. We will now show that assuming $H$, the distance between the particles exceeds $2 \rho$ at time $T_{2}$ or earlier than that. First, if $Y \in D$ on $\left[T_{1}, T_{2}\right]$, then within the time interval $\left[T_{0}, T_{2}\right]$ the mirror is fixed. Note that the angle of the mirror relative to $e_{1}$ is within $[\pi / 4, \pi / 2]$. Therefore $X_{T_{2}}$, which is inside $B_{h \rho}\left(X_{T_{1}}-5 \rho e_{1}\right)$, is at a distance of at least $5 \rho / \sqrt{2}-h \rho>\rho$ from the mirror. Hence the two particles are at a distance greater than $2 \rho$ from each other. If $Y$ hits the boundary during $\left[T_{1}, T_{2}\right]$, let

$$
U=\sup \left\{t \in\left[T_{1}, T_{2}\right]: Y_{t} \in \partial D\right\} .
$$

There are two cases: (I) If $U \in\left[T_{1}, T_{1}+3 \rho^{2}\right]$, then the mirror does not move within $\left[U, T_{2}\right]$. Recall that the angle that the mirror forms with the horizontal axis is always in the range from $\pi / 4$ to $3 \pi / 4$. It follows that on the interval $\left[U, T_{2}\right]$, the distance from $X_{T_{2}}$ to the mirror must grow to at least $2 \rho / \sqrt{2}-2 h \rho>\rho$. Hence at time $T_{2}$ the particles are more than $2 \rho$ units apart. (II) If $U \in\left(T_{1}+3 \rho^{2}, T_{2}\right.$ ], then $X_{U}$ is at a distance greater than $3 \rho / \sqrt{2}-h \rho>2 \rho$ from the boundary, while $Y_{U}$ is on the boundary, and again we find a time prior to $T_{2}$ when the particles are more than $2 \rho$ apart. We conclude that there is an $S$ satisfying (20). For a standard planar Brownian motion $W$, the probability of $\left\{\left|W_{t}-\rho^{-1} r t\right| \leq \gamma \rho, t \in\left[0, \rho^{2} T\right]\right\}$ is strictly positive by the "support theorem" and independent of $\rho$, by scaling. For $H$ to happen, two events of this form have to happen, with $X$ in place of $W$. This, the strong Markov property, and the fact that $H_{0}$ has probability greater than $1 / 2$ imply that (21) holds, with some $p>0$ that is independent of $\rho$.

Next we discuss the event $K$ which we define as

$$
K=H_{1} \cap\left\{\tau \leq T_{1}+\rho^{2}\right\} .
$$

It follows immediately from the definitions of $H_{0}$ and $H_{1}$ that on $K$ one has $\tau \leq c \rho^{2}$. If $H_{1}$ occurs, then the convex hull of $B_{h \rho}\left(X_{T_{1}}\right) \cup B_{h \rho}\left(Y_{T_{1}}\right)$ is contained in $D$. This, Brownian scaling and the strong Markov property easily imply that the probability that $\tau \leq T_{1}+\rho^{2}$ conditional on $\mathcal{F}_{T_{1}}$ is bounded below on $H_{1}$ by a positive constant independent of $\rho$. Since the probability of $H_{1}$ is itself bounded away from zero, 
(22) follows. This completes the discussion of case (a1). Case (a2) can be treated in a similar fashion, by interchanging the roles of $X$ and $Y$.

(a3) We let $H$ and $K$ be defined as in case (a1). A simple calculation shows that in this case, on $H$, the angle which $\xi_{1}$ forms with $e_{1}$ is within $[-\pi / 8, \pi / 8]$. Moreover, neither of the particles hits the boundary before time $T_{1}+\rho^{2}$, and at this time the distance between the particles is greater than $2 \rho$. Hence (20) holds true. The validity of (21) and (22) can be proved as in case (a1).

(b) In case (b), both points $x$ and $y$ must be in one of the discs $B_{128 a}\left(x^{*}\right)$ or $B_{128 a}\left(y^{*}\right)$. We will assume the latter, without loss of generality. Let $\left(\widetilde{e}_{1}, \widetilde{e}_{2}\right)$ be a coordinate system in which $D$ is locally the set of points above the graph of a Lipschitz function with constant $\lambda$. Let $H_{0}$ be defined as in case (a1). Let

$$
H=H_{0} \cap A^{X}\left(X_{T_{1}}, T_{1}, \rho^{-1} \widetilde{e}_{2}, h \rho, T_{2}\right),
$$

where $T_{1}=T_{0}, T_{2}=T_{1}+5 \lambda \rho^{2}$. Then the analysis based on the last visit of $Y$ to $\partial D$ within $\left[T_{1}, T_{2}\right]$, carried out for case (a1), applies in the current case in an analogous way and shows that there is an $S$ for which (201) holds. Again, (21) is a simple consequence of scaling.

Finally, we describe the event $K$ for case (b). Let $\overline{y^{*}}$ denote the line segment emanating from $y^{*}$ defined as $\left\{y^{*}+r \widetilde{e}_{2}: r \in[0,129 a]\right\}$. Then it is easy to see that if $x_{1} \in \overline{y^{*}}$, then for any $x \in B_{\rho / 16}\left(x_{1}\right)$, and $y \in D_{\delta \rho}$ with $y \geq x$, the convex hull of $B_{h \rho}(x) \cup B_{h \rho}(y)$ is contained in $D$. Let

$$
K_{0}=H_{0} \cap A^{X}\left(X_{T_{0}}, T_{0}, \rho^{-1} r \widetilde{e}_{1}, h \rho, \widetilde{T}_{1}\right),
$$

where $r=1$ if $\left(X_{T_{0}}-y^{*}\right) \cdot \widetilde{e}_{1}<0$, and $r=-1$ otherwise, and $\widetilde{T}_{1}$ is the first hitting time of $\overline{y^{*}}$ by $X$ after time $T_{0}$. It is easy to check that

$$
H_{0} \cap A^{X}\left(X_{T_{0}}, T_{0}, \rho^{-1} r \widetilde{e}_{1}, h \rho, T_{0}+3 \rho^{2}\right) \subset K_{0} .
$$

This and scaling show that the probability of $K_{0}$ is bounded below. Next, let $\widetilde{T}_{2}$ be defined analogously to $T_{0}$, namely,

$$
\widetilde{T}_{2}=\inf \left\{t>\widetilde{T}_{1}: X_{t}, Y_{t} \in D_{\delta \rho}, X_{t} \in B_{\rho / 16}\left(X_{\widetilde{T}_{1}}\right), Y_{t} \in B_{\rho / 16}\left(Y_{\widetilde{T}_{1}}\right)\right\} .
$$

Lemma 2 and the strong Markov property imply that conditional on $\mathcal{F}_{\widetilde{T}_{1}}$, one has $\widetilde{T}_{2} \leq \widetilde{T}_{1}+\sigma \rho^{2}$ on $\left\{\widetilde{T}_{1}<\tau\right\}$ with probability at least $1 / 2$. It follows that the event $K_{1}=K_{0} \cap\left\{\widetilde{T}_{2} \leq \widetilde{T}_{1}+\sigma \rho^{2}\right\}$ has probability bounded below by a positive constant independent of $\rho$. According to an earlier remark, under $K_{1}$, one has that the convex hull of $B_{h \rho}\left(X_{\widetilde{T}_{2}}\right) \cup B_{h \rho}\left(Y_{\widetilde{T}_{2}}\right)$ is contained in $\rho$. We conclude that

$$
K=K_{1} \cap\left\{\tau \leq \widetilde{T}_{2}+\rho^{2}\right\}
$$

has probability bounded below by a constant independent of $\rho$. This completes the proof of (22).

Let us show how (20) and (21) imply part (i) of the lemma. Let $(x, y) \in \mathcal{D}(\varepsilon)$, and let $\rho=|x-y| \geq \varepsilon$. Then

$$
P_{x, y}(T(\mathcal{D}(2 \rho))<\tau) \geq p,
$$

and by the strong Markov property,

$$
P_{x, y}\left(T\left(\mathcal{D}\left(2^{k} \rho\right)\right)<\tau\right) \geq p^{k} .
$$

Taking $k=[\log (a / \varepsilon)]+1$, we obtain

$$
P_{x, y}(T(\mathcal{D}(a))<\tau) \geq p(\varepsilon / a)^{-\log p},
$$

which completes the proof of (i). 
Consider part (ii) next. By (22), we have $P_{x, y}\left(\tau \geq c \rho^{2}\right) \leq 1-p$. By the Markov property,

$$
P_{x, y}\left(\tau \geq k c \rho^{2}\right) \leq(1-p)^{k},
$$

and therefore for any $x, y$ with $|x-y|=\rho \leq \varepsilon$,

$$
E_{x, y} T\left(\widehat{\mathcal{D}}_{\varepsilon}^{c}\right) \leq E_{x, y} \tau \leq \sum_{k \geq 1} k c \rho^{2}(1-p)^{k}=c_{1} \rho^{2} \leq c_{1} \varepsilon^{2} .
$$

Finally, we will prove part (iii) of the lemma. Suppose $t \in[u / 4, u]$ and recall $\delta$ and $\sigma$ from Lemma 2, Let $h=(\delta / 2) \wedge(1 / 16)$ and suppose that $\varepsilon_{0}>0$ is so small that $\sigma\left(2 \varepsilon_{0}\right)^{2}<u / 4$. Assume that $|x-y|=\rho \geq 2 \varepsilon_{0}$. Then Lemma 2 and the strong Markov property applied at $T_{0}$ imply that

$$
\begin{aligned}
P_{x, y} & \left(\tau>t,\left(X_{t}, Y_{t}\right) \in \mathcal{D}^{n}\left(\varepsilon_{0}\right)\right) \\
& \geq P_{x, y}\left(\tau>u,\left(X_{s}, Y_{s}\right) \in \mathcal{D}^{n}\left(\varepsilon_{0}\right), s \in[u / 4, u]\right) \\
& \geq P_{x, y}\left(T_{0} \leq \sigma\left(2 \varepsilon_{0}\right)^{2}, X_{s} \in B_{h \varepsilon_{0}}\left(X_{T_{0}}\right), Y_{s} \in B_{h \varepsilon_{0}}\left(Y_{T_{0}}\right), s \in\left[T_{0}, T_{0}+u\right]\right) \\
& \geq(1 / 2) P_{x}\left(W_{s} \in B_{h \varepsilon_{0}}(x), s \in[0, u]\right) \\
& \geq c .
\end{aligned}
$$

Here $W$ denotes a planar Brownian motion starting from $x$. The third inequality follows from the fact that $X$ and $Y$ do not visit the boundary within $\left[T_{0}, T_{0}+u\right]$, if the event on the third line holds, because of the definition of $T_{0}$ given in Lemma 2

The following lemma is almost the same as Lemma 5.1 in [4]. We reproduce it here because it is one of the most important elements of our argument. The present form of the result is very close to Lemma 3.10 in [9]. The intuitive meaning of the lemma is that if we condition $X$ and $Y$ on not coupling before time $u>0$, then the processes are likely to move apart for a considerable distance at time $u$. Hence, Lemma 4 below is a version of the parabolic boundary Harnack principle for the process $(X, Y)$.

Lemma 4. For every $u>0$ there exist constants $c_{1}, c_{2}>0$ such that for all $n$ large and for all $x, y \in D^{n}$ with $x \geq y$ and $x \neq y$,

$$
P_{x, y}^{n}\left(\left|X_{u}-Y_{u}\right|>c_{1} \mid \tau>u\right)>c_{2} .
$$

Proof. The proof of this lemma uses methods considerably different from those in the rest of the paper so it will be convenient to introduce some notation specific only to this proof. First of all recall the following convention from the proof of Lemma 3- the superscript $n$ will be dropped from all pieces of notation. This should not cause any confusion because the constants in Lemma 3 do not depend on $n$.

We will write $Z=(X, Y)$ since the separate components of $Z$ will play no role in this proof. The state space of $Z$ is $\bar{D} \times \bar{D}$. Recall $\mathcal{D}, \mathcal{D}(\varepsilon)$ and $\widehat{\mathcal{D}}(\varepsilon)$ defined before Lemma 3 The distribution of $Z$ starting from $z$ and the corresponding expectation will be denoted $P^{z}$ and $E^{z}$. Conditioning by a harmonic function $h$ will be reflected in the notation by writing $P_{h}^{z}$ and $E_{h}^{z}$. See [12] for the discussion of conditioned Brownian motion and [18] for conditioning of general Markov processes.

We will denote the space-time counterpart of $Z$ by $V$. More precisely, if $Z$ has law $P^{z}$, then the law of the space-time process $\left\{V_{t}=\left(Z_{t}, s-t\right), t \geq 0\right\}$ will be denoted $P^{z, s}$. The distribution of the space-time process conditioned by a parabolic 
function $g$ will be denoted $P_{g}^{z, s}$. By abuse of notation, $T(A)$ will denote the first hitting time of $A$ for $V$ as well as for $Z$.

Fix some small $\varepsilon_{0}>0$ such that Lemma 3 applies with $\varepsilon_{0}$, and let $M=\mathcal{D}\left(\varepsilon_{0}\right)$ and $\mathcal{D}_{1}=\widehat{\mathcal{D}}\left(2 \varepsilon_{0}\right)$. Let $h(z)=P^{z}\left(T\left(\mathcal{D}\left(2 \varepsilon_{0}\right)\right)<\tau\right)$ and $U_{k}=\left\{z \in \mathcal{D}_{1}: h(z) \in\left[2^{k-1}, 2^{k}\right]\right\}$ for integer $k$.

By Lemma 3 (i), $U_{k} \subset \widehat{\mathcal{D}}\left(c_{1} 2^{k / \alpha}\right)$, for some $c_{1}, \alpha>0$. Then Lemma 3 (ii) shows that $\sup _{z \in U_{k}} E^{z}\left(T\left(U_{k}^{c}\right)\right) \leq c_{2} 2^{2 k / \alpha}$. It follows that

$$
\sum_{k=0}^{\infty} \sup _{z \in U_{-k}} E^{z}\left(T\left(U_{-k}^{c}\right)\right)<\infty
$$

An argument of Chung [1] (see also 4]) shows that for suitable $c_{3}$,

$$
c_{3} \sum_{k=0}^{\infty} \sup _{z \in U_{-k}} E^{z}\left(T\left(U_{-k}^{c}\right)\right)
$$

is an upper bound for $E_{h}^{z}\left(T\left(\mathcal{D}_{1}^{c}\right)\right)$. It follows that for a suitable $c_{4}>0$ and every $z \in \mathcal{D}$

$$
P_{h}^{z}\left(T\left(\mathcal{D}_{1}^{c}\right)<u / 4\right)>c_{4} .
$$

Recall the discussion of space-time processes at the beginning of the proof. The function

$$
(z, t) \mapsto g(z, t)=P^{z}(\tau>t)
$$

is parabolic in $\mathcal{D} \times[0, \infty)$ with boundary values 1 on $\mathcal{D} \times\{0\}$ and 0 otherwise. Let $g_{1}$ be a parabolic function in $\mathcal{D} \times[0, \infty)$ which has the same boundary values as $g$ except that $g_{1}(z, 0)$ is changed from 1 to $\delta$ for $z \in \mathcal{D} \backslash M$, where $\delta \in(0,1)$ will be chosen later.

We will use the following notation convention in the next displayed formula. Suppose that $V_{t}=\left(Z_{t}, s-t\right)$ has the distribution $P^{x, s}$. Then $T_{*}\left(\mathcal{D}_{1}^{c}\right)=s-$ $\inf \left\{t>0: V_{t} \in \mathcal{D}_{1}^{c} \times \mathbb{R}\right\}$. Note that, typically, $T_{*}\left(\mathcal{D}_{1}^{c}\right)<s$.

By Lemma 3 (iii), we have $g_{1}(z, s)>c_{5}$ for all $z \in \mathcal{D}\left(2 \varepsilon_{0}\right)$ and $s \in[u / 4, u]$, where $c_{5}$ does not depend on $\delta$. We obviously have $h(y) \leq 1$ for all $y$. Let $h(x, s)=h(x)$. For $x \in \mathcal{D}_{1}$ and $s \geq u / 2$ we have, by (24),

$$
\begin{aligned}
g_{1}(x, s) & \geq \int_{\substack{t \in[u \mid 4, s] \\
y \in \partial \mathcal{D}\left(2 \varepsilon_{0}\right)}} g_{1}(y, t) P^{x, s}\left(T_{*}\left(\mathcal{D}_{1}^{c}\right) \in d t, Z\left(T_{*}\left(\mathcal{D}_{1}^{c}\right)\right) \in d y\right) \\
& =\int_{\substack{t \in[u \mid 4, s] \\
y \in \partial \mathcal{D}\left(2 \varepsilon_{0}\right)}} \frac{h(x, s)}{h(y, t)} \frac{h(y, t)}{h(x, s)} g_{1}(y, t) P^{x, s}\left(T_{*}\left(\mathcal{D}_{1}^{c}\right) \in d t, Z\left(T_{*}\left(\mathcal{D}_{1}^{c}\right)\right) \in d y\right) \\
& =\int_{\substack{t \in[u \mid 4, s] \\
y \in \partial \mathcal{D}\left(2 \varepsilon_{0}\right)}} \frac{h(x, s)}{h(y, t)} g_{1}(y, t) P_{h}^{x, s}\left(T_{*}\left(\mathcal{D}_{1}^{c}\right) \in d t, Z\left(T_{*}\left(\mathcal{D}_{1}^{c}\right)\right) \in d y\right) \\
& \geq \int_{\substack{t \in[u \mid 4, s] \\
y \in \partial \mathcal{D}\left(22_{0}\right)}} h(x, s) c_{5} P_{h}^{x, s}\left(T_{*}\left(\mathcal{D}_{1}^{c}\right) \in d t, Z\left(T_{*}\left(\mathcal{D}_{1}^{c}\right)\right) \in d y\right) \\
& =h(x, s) c_{5} P_{h}^{x, s}\left(T_{*}\left(\mathcal{D}_{1}^{c}\right) \in[u / 4, s]\right) \\
& \geq h(x, s) c_{5} c_{4}=c_{6} h(x, s)=c_{6} h(x) .
\end{aligned}
$$


Let $U_{k}^{*}=\left\{z \in \mathcal{D}_{1}: h(z) \leq 2^{k}\right\}$ for integer $k$. We argue as in the case of $U_{k}$ 's. By Lemma 3 (i), $U_{k}^{*} \subset \widehat{\mathcal{D}}\left(c_{1} 2^{k / \alpha}\right)$, for some $c_{1}, \alpha>0$. Lemma 3 (ii) shows that $\sup _{z \in U_{k}^{*}} E^{z}\left(T\left(\left(U_{k}^{*}\right)^{c}\right)\right) \leq c_{2} 2^{2 k / \alpha}$. It follows that

$$
\sum_{k=0}^{\infty} \sup _{z \in U_{-k}^{*}} E^{z}\left(T\left(\left(U_{-k}^{*}\right)^{c}\right)\right)<\infty .
$$

Let

$$
\begin{gathered}
W_{k}=\left\{(z, s): g_{1}(z, s) \in\left[2^{k}, 2^{k+1}\right], s \in[u / 2, u]\right\}, \\
W=\bigcup_{k=-\infty}^{k_{1}} W_{k},
\end{gathered}
$$

where $k_{1}<0$ will be chosen later. If $2^{-m}<c_{6}$, then $W_{k} \subset U_{k+m}^{*} \times[u / 2, u]$. Let such $m$ be fixed. Using the estimate of Chung [11], we obtain for small $k_{1}$ and all $z \in \mathcal{D}$,

$$
\begin{aligned}
E_{g_{1}}^{z, u}\left(T\left(W^{c}\right)\right) & \leq \quad c_{7} \sum_{k=-\infty}^{k_{1}} \sup _{(y, s) \in W_{k}} E^{y, s} T\left(W_{k}^{c}\right) \\
& \leq \quad c_{7} \sum_{k=-\infty}^{k_{1}} \sup _{y \in U_{k+m}^{*}} E^{y} T\left(\left(U_{k+m}^{*}\right)^{c}\right) \\
& \leq \quad c_{2} c_{7} \sum_{k=-\infty}^{k_{1}} 2^{2(k+m) / \alpha}=: R\left(k_{1}\right)<\infty
\end{aligned}
$$

Choose $k_{1}$ so small that $R\left(k_{1}\right)<u / 8$, and note that $k_{1}$ is independent of $n$ and $\delta$. Then for any $z \in \mathcal{D}$,

$$
E_{g_{1}}^{z, u} T\left(W^{c}\right)<u / 8 .
$$

Let

$$
Q=\left\{(x, s): g_{1}(x, s) \geq 2^{k_{1}}, s \in[u / 2, u]\right\} .
$$

Since the $g_{1}$-process cannot exit $\mathcal{D} \times[0, \infty)$ through $\partial \mathcal{D} \times[0, \infty)$, 25) implies

$$
P_{g_{1}}^{z, u}(T(Q)>u / 4)<1 / 2 .
$$

Now let $\delta=2^{k_{1}-1}$. Since $0 \leq g_{1} \leq 1$, the process $g_{1}\left(V_{t}\right)$ is a martingale under $P^{z, s}$, and $g_{1}(z, s) \geq 2^{k_{1}}$ for $(z, s) \in Q$, we see that there is at least a $2^{k_{1}-1} / 2$ chance that $V$ under $P^{z, s}$ will hit $M \times\{0\}$ before hitting any other part of $\partial(\mathcal{D} \times[0, \infty))$. Thus we have for $(z, s) \in Q$,

$$
\begin{aligned}
P_{g_{1}}^{z, s}\left[V_{s} \in M \times\{0\}\right] & =\int_{M}\left(g_{1}(y, 0) / g_{1}(z, s)\right) P^{z, s}\left[V_{s} \in d y, T(\partial(\mathcal{D} \times[0, \infty)))=s\right] \\
& \geq \int_{M} P^{z, s}\left[V_{s} \in d y, T(\partial(\mathcal{D} \times[0, \infty)))=s\right] \\
& \geq 2^{k_{1}-1} / 2 .
\end{aligned}
$$

This and (26) yield, by the strong Markov property, for all $z \in \mathcal{D}$,

$$
P_{g_{1}}^{z, u}\left[V_{u} \in M \times\{0\}\right] \geq c_{8}>0 .
$$


The ratio of $g$ and $g_{1}$ is bounded away from 0 and $\infty$ on the accessible boundary of $\mathcal{D} \times[0, \infty)$, so

$$
P_{g}^{z, u}\left[V_{u} \in M \times\{0\}\right] \geq c_{9}>0
$$

for all $z \in \mathcal{D}$. This is equivalent to the statement in the lemma.

\section{NeUMANN EIGENFUnCTIONS}

We will consider lip domains $D \subset \mathbb{R}^{2}$ in this section.

Recall the coordinate systems $\left(e_{1}, e_{2}\right)$ and $\left(e_{1}^{\prime}, e_{2}^{\prime}\right)$, and the partial order " $\leq$ " on $\mathbb{R}^{2}$, defined before Proposition 2, For a function $u \in C^{1}(D)$ write

$$
\partial^{\prime} u=\min \left\{\frac{\partial u}{\partial e_{1}^{\prime}}, \frac{\partial u}{\partial e_{2}^{\prime}}\right\} .
$$

Let

$$
\begin{aligned}
& S=\left\{u \in C^{1}(D): \partial^{\prime} u(x) \geq 0, x \in D\right\}, \\
& \widetilde{S}=\left\{u \in C^{1}(D): \partial^{\prime} u(x)>0, x \in D\right\} .
\end{aligned}
$$

Lemma 5. Let $D$ be a lip domain other than a rectangle. Consider a Neumann eigenfunction $\psi$ in $D$ corresponding to the second eigenvalue. If $\psi \in S$, then $\psi \in \widetilde{S}$.

Proof. We will first prove the following claim.

(A) There exists a nonempty ball $B \subset D$ and a constant $a>0$ such that $\partial^{\prime} \psi \geq a$ on $B$.

Assume that (A) does not hold. Since $\psi \in S$ and $\psi$ is nonconstant, there is $x \in D$ where $\partial \psi(x) / \partial e_{i}^{\prime}>0$ for either $i=1,2$. Assume without loss of generality that $\partial \psi(x) / \partial e_{1}^{\prime}>0$. The inequality holds in a neighborhood of $x$, by continuity of $\nabla \psi$. We have assumed that (A) fails, so there exists an open set where $\partial \psi / \partial e_{2}^{\prime}=0$. Since $\psi$, as an eigenfunction, is real-analytic in $D, \partial \psi / \partial e_{2}^{\prime}=0$ holds everywhere in $D$. Hence $\psi(x)=g\left(x_{1}\right)$, where $x_{1}$ refers to the first coordinate of $x=\left(x_{1}, x_{2}\right)$ in the coordinate system $\left(e_{1}^{\prime}, e_{2}^{\prime}\right)$. The only function of the form $\psi(x)=g\left(x_{1}\right)$ which satisfies the equation $\Delta \psi=-\mu_{2} \psi$ is $\psi(x)=\cos x_{1}$, upon appropriate translation and scaling of the coordinate system. As a generalized solution to the eigenfunction equation, $\psi$ satisfies for any $f \in C^{2}(D)$,

$$
\mu_{2} \int_{D} f \psi d x+\int_{D} \psi \Delta f d x+\int_{\partial D} \psi \frac{\partial f}{\partial \nu} \sigma(d x)=0
$$

where $\nu$ is the inward unit normal vector field, and $\sigma$ is the surface measure on $\partial D$. The divergence theorem implies that

$$
\mu_{2} \int_{D} f \psi d x=\int_{D} \nabla f \cdot \nabla \psi d x
$$

We will use a test function of the form $f(x)=\theta\left(x_{2}\right)$. For any such function $f$, the right-hand side of the last formula is zero, and so we have

$$
\int_{D} \cos x_{1} \theta\left(x_{2}\right) d x=0
$$

The eigenfunction $\psi$ must vanish at some points of $D$. It follows from the periodicity of $\psi(x)=\cos x_{1}$ that we can assume without loss of generality that $\psi$ vanishes on $\left\{x \in D: x_{1}=\pi / 2\right\}$. By Courant's Nodal Line Theorem, $\psi$ cannot vanish anywhere else in $D$. Hence, $D \subset\left\{x:-\pi / 2<x_{1}<3 \pi / 2\right\}$. Recall from Section $\square$ the left and right vertices of $D$, called $x^{*}$ and $y^{*}$. Let $\alpha_{1}\left[\right.$ resp., $\left.\alpha_{2}\right]$ denote the line through $x^{*}$ 
[resp., $\left.y^{*}\right]$, parallel to the $e_{1}^{\prime}$-axis. The fact that $D$ is a lip domain which is not a rectangle implies that the projection onto the $e_{1}^{\prime}$-axis of $\alpha_{1} \cap \partial D$ is not equal to that of $\alpha_{2} \cap \partial D$. Hence, for either $i=1$ or $2, \alpha_{i} \cap \partial D$ is not symmetric about the line through the point $(\pi / 2,0)$ parallel to $e_{2}^{\prime}$. Assume without loss of generality that there is no symmetry for $i=1$. Then either $\int_{\alpha \cap D} \psi\left(x_{1}, x_{2}\right) d x_{1}>0$ for all lines $\alpha$ which intersect $D$ and are sufficiently close to $\alpha_{1}$ or the integral is strictly negative for all such $\alpha$. Now let $\theta$ be such that $f(x)=\theta\left(x_{2}\right)$ is zero off an $\varepsilon$-neighborhood of $\alpha_{1}$, it is equal to 1 in a neighborhood of $\alpha_{1}$, and it is nonnegative in $D$. Then, if $\varepsilon$ is small enough, $\int_{D} f \psi d x \neq 0$. This contradicts [27), and therefore (A) holds.

We will need the following result from 8 (Theorem 2.4) on "synchronous couplings." Suppose $x, y \in D$. Then there is a probability space $\left(\Omega^{\prime}, \mathcal{F}^{\prime}, P_{x, y}^{\prime}\right)$ and a process $(W, X, Y)$ such that $X$ [respectively, $Y$ ] is a reflected Brownian motion in $\bar{D}$ starting from $x[y]$ and $W$ is a Brownian motion on the filtration generated by $(W, X, Y)$, such that $(X, Y)$ admits the following Skorohod representation:

$$
X_{t}=x+W_{t}+\int_{0}^{t} \nu\left(X_{s}\right) d|L|_{s}, \quad Y_{t}=y+W_{t}+\int_{0}^{t} \nu\left(Y_{s}\right) d|M|_{s},
$$

where $|L|$ and $|M|$ are boundary local times for $X$ and $Y$, respectively, and where $\nu$ is the inward normal vector field on $\partial D$. Theorem 2.4 of $[8]$ shows that the synchronous coupling $(X, Y)$ in $D$ may be obtained as a limit of synchronous couplings in a sequence of polygonal lip domains $D_{k}$ approximating $D$. For polygonal lip domains, the following "order preserving property" has been proved in 3], and so it holds for the coupling $(X, Y)$ in $D$; if $x \leq y$, then $X_{t} \leq Y_{t}$ for all $t \geq 0, P_{x, y}^{\prime}$-a.s. We have for any $t>0$,

$$
e^{-\mu_{2} t} \psi(x)=E_{x, y}^{\prime} \psi\left(X_{t}\right), \quad e^{-\mu_{2} t} \psi(y)=E_{x, y}^{\prime} \psi\left(Y_{t}\right) .
$$

Let $B=B_{\rho}\left(x_{0}\right)$ be a ball satisfying claim (A) proved at the beginning of the proof, and let $\widetilde{B}=B_{\rho / 2}\left(x_{0}\right)$. Let $x \in D, y=x+r e_{1}^{\prime}$ and $T=\inf \left\{t: \operatorname{dist}\left(X_{t}, \partial D\right)<\right.$ $\operatorname{dist}(x, \partial D) / 2\}$. Note that $\psi\left(Y_{1}\right)-\psi\left(X_{1}\right) \geq 0$ with probability 1 , because $X_{1} \leq Y_{1}$ and $\psi \in S$. We have $Y_{1}-X_{1}=Y_{0}-X_{0}$ if $T>1$. Hence for all $r \in(0, \rho / 2)$ such that $y \in D$,

$$
\begin{aligned}
r^{-1}(\psi(y)-\psi(x)) & \geq r^{-1} e^{\mu_{2}} E_{x, y}^{\prime}\left[\left(\psi\left(Y_{1}\right)-\psi\left(X_{1}\right)\right) 1_{\left\{X_{1} \in \tilde{B}, T>1\right\}}\right] \\
& \geq\left(e^{\mu_{2}} a / 2\right) P_{x, y}^{\prime}\left(X_{1} \in \widetilde{B}, T>1\right),
\end{aligned}
$$

where $a>0$ is the constant in (A). Since the right-hand side is positive and independent of $r$, it follows that $\partial \psi(x) / \partial e_{1}^{\prime}>0$. A similar argument applies to $\partial \psi / \partial e_{2}^{\prime}$, and we conclude that $\partial^{\prime} \psi>0$ on $D$.

Recall that $D_{\varepsilon}=\{x \in D: \operatorname{dist}(x, \partial D) \geq \varepsilon\}$.

Lemma 6. Suppose $D$ is a lip domain other than a rectangle. For any $\varepsilon_{1}>0$ such that the interior of $D_{\varepsilon_{1}}$ is nonempty, and any $\delta, b>0$, there exists $\varepsilon_{2}>0$ with the following property. If $\psi$ is a Neumann eigenfunction corresponding to $\mu_{2}$ with

$$
\begin{cases}\partial^{\prime} \psi \geq \delta & \text { on } D_{\varepsilon_{1}}, \\ \partial^{\prime} \psi \geq 0 & \text { on } D_{\varepsilon_{2}}, \\ |\psi| \leq b & \text { on } D\end{cases}
$$


then

$$
\partial^{\prime} \psi \geq 0 \quad \text { on } D
$$

Proof. Let $E_{x}$ be the expectation corresponding to the distribution of reflected Brownian motion in $D$, starting from $x$. Then for any $t \geq 0$, and $x, y \in \bar{D}$,

$$
e^{-\mu_{2} t}(\psi(x)-\psi(y))=E_{x} \psi\left(X_{t}\right)-E_{y} \psi\left(Y_{t}\right) .
$$

Hence, it will suffice to show that for all $x, y \in D, x \geq y$,

$$
E_{x} \psi\left(X_{2}\right)-E_{y} \psi\left(Y_{2}\right) \geq 0 .
$$

Recall the domains $D^{n}$ from Section 3. We will denote by $P_{x, y}^{n}$ a measure under which $(X, Y)$ is a pair of reflected Brownian motions in $D^{n}$, starting from $(x, y)$, which is mirror coupled on the time interval $[0,1)$ and synchronously coupled on $[1,2]$ (see the proof of Lemma 5 for the definition of a synchronous coupling).

If $x \in D$, then the distributions of $X$ under $P_{x, y}^{n}$ converge weakly to the distribution of the reflected Brownian motion in $D$ in the uniform topology on $C\left([0,2], \mathbb{R}^{2}\right)$, as $n \rightarrow \infty$, by Theorem 2 of [7]. Since a similar remark applies to $Y$, it is enough to show that there exists $\varepsilon_{2}>0$ such that for all large $n$,

$$
E_{x, y}^{n}\left(\psi\left(X_{2}\right)-\psi\left(Y_{2}\right)\right) \geq 0
$$

Denote the coupling time by $\tau$. Since $X_{2}=Y_{2}$ on $\{\tau \leq 1\}$, it suffices to show that

$$
E_{x, y}^{n}\left[\psi\left(X_{2}\right)-\psi\left(Y_{2}\right) \mid \tau>1\right] \geq 0 .
$$

By the uniform continuity of transition probabilities in $D^{n}$ 's proved in Theorem 2.1 of $[8]$,

$$
P_{x, y}^{n}\left(X_{1} \in C\right) \leq c|C|
$$

for any Borel subset $C$ of $\bar{D}$, where $|C|$ denotes the Lebesgue measure of $C$ and $c$ does not depend on $x, C$ and $n$. Let $c_{1}$ and $c_{2}$ be the constants from the statement of Lemma 4. We can assume that $\varepsilon_{2}<\varepsilon_{1}$. By Proposition 2 $X_{2} \geq Y_{2}$ a.s. Recall that $\partial^{\prime} \psi \geq 0$ on $D_{\varepsilon_{2}}$. This implies that $\psi\left(X_{2}\right)-\psi\left(Y_{2}\right) \geq 0$ on $\left\{X_{2}, Y_{2} \in D_{\varepsilon_{2}}\right\}$. By (29),

$$
\begin{aligned}
E_{x, y}^{n}( & \left.\psi\left(X_{2}\right)-\psi\left(Y_{2}\right) \mid \tau>1\right) \\
\geq & E_{x, y}^{n}\left[\left(\psi\left(X_{2}\right)-\psi\left(Y_{2}\right)\right) 1_{\left\{X_{2}, Y_{2} \in D_{\varepsilon_{2}}\right\}} \mid \tau>1\right] \\
& -2 b\left[P_{x, y}\left(X_{2} \notin D_{\varepsilon_{2}} \mid \tau>1\right)+P_{x, y}\left(Y_{2} \notin D_{\varepsilon_{2}} \mid \tau>1\right)\right] \\
\geq & E_{x, y}^{n}\left[\left(\psi\left(X_{2}\right)-\psi\left(Y_{2}\right)\right) 1_{\left\{X_{2}, Y_{2} \in D_{\varepsilon_{1}},\left|X_{2}-Y_{2}\right|>c_{1} / 2\right\}} \mid \tau>1\right]-c \varepsilon_{2} .
\end{aligned}
$$

Since $\partial^{\prime} \psi \geq \delta$ on $D_{\varepsilon_{1}}$, we have $\psi\left(X_{2}\right)-\psi\left(Y_{2}\right) \geq \delta c_{1} / 2$ on $\left\{X_{2}, Y_{2} \in D_{\varepsilon_{1}},\left|X_{2}-Y_{2}\right|>\right.$ $\left.c_{1} / 2\right\}$, so

$$
E_{x, y}^{n}\left(\psi\left(X_{2}\right)-\psi\left(Y_{2}\right) \mid \tau>1\right) \geq c P_{x, y}^{n}\left(X_{2}, Y_{2} \in D_{\varepsilon_{1}},\left|X_{2}-Y_{2}\right|>c_{1} / 2 \mid \tau>1\right)-c \varepsilon_{2} .
$$

It is clear that Lemma 4 remains valid if we replace $c_{1}$ in that lemma with any smaller constant. Assume that $c_{1}>0$ is so small that there exists $z \in D$ such that $B_{2 c_{1}}(z) \subset D_{\varepsilon_{1}}$. An easy argument based on Lemma 2 and the strong Markov property shows that if $\left|X_{1}-Y_{1}\right|>c_{1}$, then with some probability $p>0$, independent of $n$, we have $X_{2}, Y_{2} \in D_{\varepsilon_{1}}$ and $\left|X_{2}-Y_{2}\right|>c_{1} / 2$. Hence,

$$
\begin{aligned}
E_{x, y}^{n}\left(\psi\left(X_{2}\right)-\psi\left(Y_{2}\right) \mid \tau>1\right) & \geq c P_{x, y}^{n}\left[\left|X_{1}-Y_{1}\right|>c_{1} \mid \tau>1\right]-c \varepsilon_{2} \\
& \geq c c_{2}-c \varepsilon_{2},
\end{aligned}
$$


where the last inequality follows from Lemma 4 Note that $c_{2}$ and the other constants in the last formula do not depend on $x$ and $y$. Taking $\varepsilon_{2}>0$ small, (28) follows, and Lemma 6 is proved.

Proof of Theorem 1 . One can easily verify that both parts of the theorem hold in rectangles so we will assume that $D$ is not a rectangle.

(i) The argument is similar to that in the proof of Theorem 4.1 in [2]. Arguing by contradiction, assume that $\mu_{2}$ is multiple.

Recall from the proof of Lemma 5 that there exists a synchronous coupling of reflecting Brownian motions in $D$ with the property that $X_{t} \leq Y_{t}$ for all $t \geq 0$, if $X_{0} \leq Y_{0}$. This and the argument given in the proof of Theorem 3.3 of 3 ] show that at least one of the eigenfunctions corresponding to $\mu_{2}$ belongs to $S$. Fix one of these eigenfunctions and call it $\phi$.

We have assumed that $\mu_{2}$ is multiple so there exists an eigenfunction $\phi^{\perp}$ which is orthogonal to $\phi$. Since $\phi^{\perp}$ and $-\phi^{\perp}$ cannot both be in $S$, we can assume without loss of generality that $\phi^{\perp} \notin S$. Let

$$
\phi^{a}=(1-a) \phi+a \phi^{\perp}, \quad a \in[0,1],
$$

and

$$
a^{*}=\inf \left\{a \in[0,1]: \phi^{a} \notin S\right\} .
$$

We claim that $a^{*}<1$. Since $\partial^{\prime} \phi^{a} \geq 0$ on $D$ for $a<a^{*}$ and $\partial^{\prime} \phi^{a} \rightarrow \partial^{\prime} \phi^{a^{*}}$ pointwise in $D$ when $a \uparrow a^{*}$, we have that $\phi^{a^{*}} \in S$. Hence $a^{*}$ cannot be equal to 1 . Therefore there is a sequence $a_{k}$ with

$$
a_{k} \downarrow a^{*}, \quad a_{k} \in\left(a^{*}, 1\right), \quad \phi^{a_{k}} \notin S .
$$

For $a \in\left(a^{*}, 1\right)$ let

$$
\varepsilon(a)=\sup \left\{\operatorname{dist}(x, \partial D): \partial^{\prime} \phi^{a}(x)<0\right\} .
$$

Note that as $a \downarrow a^{*}, \partial^{\prime}\left(\phi^{a}\right) \rightarrow \partial^{\prime}\left(\phi^{a^{*}}\right)$ uniformly on compact subsets of $D$. Since

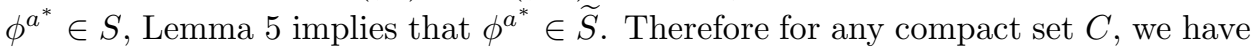
$\partial^{\prime}\left(\phi^{a}\right)>0$ on $C$ for all $a$ sufficiently close to $a^{*}$. Hence

$$
\lim _{a \downarrow a^{*}} \varepsilon(a)=0 .
$$

Let $\varepsilon_{1}>0$ be as in Lemma 6 Using again the facts that the convergence $\partial^{\prime} \phi^{a} \rightarrow$ $\partial^{\prime} \phi^{a^{*}}$ is uniform on compacts and that $\phi^{a^{*}} \in \widetilde{S}$, we have that there are constants $\delta>0$ and $a_{1} \in\left(a^{*}, 1\right)$ such that $\partial^{\prime} \phi^{a} \geq \delta$ on $D_{\varepsilon_{1}}$ for all $a \in\left(a^{*}, a_{1}\right)$. Since by definition, $\partial^{\prime} \phi^{a} \geq 0$ on $D_{\varepsilon(a)}$ for all $a \in\left(a^{*}, a_{1}\right)$, Lemma [6] and (31) show that $\phi^{a} \in S$ for all $a \in\left(a^{*}, a_{2}\right)$, where $a_{2} \in\left(a^{*}, a_{1}\right)$. This gives a contradiction to (30). As a result, the eigenvalue is simple, and Theorem 1 (i) follows.

(ii) We have shown in the first part of the proof that there is only one eigenfunction corresponding to $\mu_{2}$ and that it belongs to $S$. This immediately implies part (ii) of the theorem.

\section{ACKNOWLEDGMENTS}

We would like to express our gratitude to the following colleagues for very useful advice: R. Bañuelos, R. Bass, Z. Chen, S. Jacka, D. Jerison, C. Kenig, J.-B. Lasserre, T. Pakes, P. Pollett, and R. Tweedie. 


\section{REFERENCES}

[1] O. Adelman, K. Burdzy and R. Pemantle (1998). Sets avoided by Brownian motion. Ann. Probab. 26, 429-464. MR 99i:60152

[2] R. Atar (2001). Invariant wedges for a two-point reflecting Brownian motion and the "hot spots" problem. Elect. J. of Probab. 6, paper 18, 1-19. MR 2002i:60146

[3] R. Bañuelos and K. Burdzy (1999). On the "hot spots" conjecture of J. Rauch. J. Funct. Anal. 164, 1-33. MR 2000m:35085

[4] R. Bass and K. Burdzy (1992). Lifetimes of conditioned diffusions. Probab. Theory Related Fields 91, 405-443. MR 93e:60155

[5] R. Bass and K. Burdzy (2000). Fiber Brownian motion and the 'hot spots' problem. Duke Math. J. 105, 25-58. MR 2001g:60190

[6] R. F. Bass and E. P. Hsu (1991). Some potential theory for reflecting Brownian motion in Hölder and Lipschitz domains. Ann. Probab. 19, 486-508. MR 92i:60142

[7] K. Burdzy and Z.-Q. Chen (1998). Weak convergence of reflecting Brownian motions. Elect. Comm. in Probab. 3, 29-33. MR 99d:60091

[8] K. Burdzy and Z.-Q. Chen (2002). Coalescence of synchronous couplings. Probab. Theory Related Fields 123, 553-578. MR 2003e:60184

[9] K. Burdzy and W. S. Kendall (2000). Efficient Markovian couplings: examples and counterexamples. Ann. Appl. Probab. 10, 362-409. MR 2002b:60129

[10] K. Burdzy and W. Werner (1999). A counterexample to the "hot spots" conjecture. Ann. Math. 149, 309-317. MR 2000b:35044

[11] K. L. Chung (1984). The lifetime of conditioned Brownian motion in the plane. Ann. Inst. Henri Poincaré 20, 349-351. MR 86d:60088

[12] J. L. Doob. Classical Potential Theory and Its Probabilistic Counterpart. Springer-Verlag, New York, 1984. MR 85k:31001

[13] R. Hempel, L. A. Seco and B. Simon (1991). The essential spectrum of Neumann Laplacians on some bounded singular domains. J. Func. Anal. 102, 448-483. MR 93h:35144

[14] D. Jerison and N. Nadirashvili (2000). The "hot spots" conjecture for domains with two axes of symmetry. J. Amer. Math. Soc. 13, 741-772. MR 2001f:35110

[15] I. Karatzas and S. E. Shreve. Brownian Motion and Stochastic Calculus, second edition, Springer, New-York, 1991. MR 92h:60127

[16] B. Kawohl. Rearrangements and Convexity of Level Sets in PDE. Lecture Notes in Mathematics 1150, Springer, Berlin, 1985. MR 87a:35001

[17] W. S. Kendall (1989). Coupled Brownian motions and partial domain monotonicity for the Neumann heat kernel. J. Funct. Anal. 86, 226-236. MR 90m:58203

[18] M. Sharpe. General Theory of Markov Processes. Academic Press, New York, 1989. MR 89m:60169

[19] P. L. Lions and A. S. Sznitman (1984). Stochastic differential equations with reflecting boundary conditions. Comm. Pure appl. Math. 37, 511-537. MR 85m:60105

[20] M. Pascu (2002). Scaling coupling of reflecting Brownian motions and the hot spots problem. Trans. Amer. Math. Soc. 354, 4681-4702. MR 2003i:60141

[21] P. Protter. Stochastic Integration and Differential Equations. A New Approach. SpringerVerlag, Berlin (1990). MR 91i:60148

[22] F.-Y. Wang (1994). Application of coupling methods to the Neumann eigenvalue problem. Probab. Theory Related Fields 98, 299-306. MR 94k:58153

Department of Electrical Engineering, Technion-Israel Institute of Technology, HAIFA 32000, ISRAEL

E-mail address: atar@ee.technion.ac.il

Department of Mathematics, University of Washington, Seattle, Washington 981954350

E-mail address: burdzy@math.washington.edu 